\title{
Lattice duality for coupling pairs admitting polytope duality with trivial toric contribution
}

\section{Makiko Mase $^{1}$ (D)}

Received: 25 October 2019 / Accepted: 11 August 2021 / Published online: 20 August 2021

(c) The Author(s) 2021

\begin{abstract}
We study a lattice duality among families of $K 3$ surfaces associated to coupling pairs that admit polytope duality with trivial toric contribution.
\end{abstract}

Keywords Families of $K 3$ surfaces Coupling of weight systems Duality of Picard lattices - Toric hypersurfaces determined by lattice polytopes

Mathematics Subject Classification $14 \mathrm{~J} 28 \cdot 14 \mathrm{~J} 17 \cdot 14 \mathrm{C} 22 \cdot 52 \mathrm{~B} 20$

\section{Introduction}

Weight systems appear in many interesting spots in algebraic geometry including singularity theory, where singularities have nice properties. We focus on a duality among weight systems called coupling introduced by Ebeling (2006), which is for wellposed weight systems associated to simple $K 3$ singularities classified by Yonemura (1990). The coupling duality is in particular admitted by a pair of singularities defined by weighted-homogeneous polynomials $f$ and $f^{\prime}$ as a strange-duality for invertible polynomials introduced by Ebeling and Takahashi (2011). It is also known that such polynomials $f$ and $f^{\prime}$ in three variables can be projectivised as weighted-homogeneous polynomials $F$ and $F^{\prime}$ as anticanonical divisor of the weighted projective spaces $\mathbb{P}_{a}$ and $\mathbb{P}_{b}$, where the pair $(a, b)$ is coupling among Yonemura's list. Since all the weighted projective spaces with weights being in Yonemura's list are Fano, we obtain subfamilies of $K 3$ surfaces in the space once one finds a reflexive polytope as a subpolytope of the defining polytope of the space. In the author's recent work (Mase 2021), an existence and duality of such reflexive polytopes are studied and it is concluded that almost all coupling pair extends to a polytope-duality. Once one obtains families of $K 3$ surfaces which already admit several dualities, one may be interested in intrinsic

Makiko Mase

mmase@mail.uni-mannheim.de

1 Universität Mannheim, Lehrstuhl für Mathematik VI B6 26, 68131 Mannheim, Germany 
properties of $K 3$ surfaces. We are interested in lattice-duality originally studied by Dolgachev (1996). It is concluded by Mase $(2015,2017)$ that a part of transpose-dual pairs associated to strange duality of bimodal singularities extends to lattice dual, and that some subfamilies of $K 3$ surfaces that are double covering of the projective plane have lattice-dual property as is studied in Mase (2021). In this paper, focusing on polytope-dual pairs associated to coupling, one may pose the following problem.

PROBLEM Determine whether or not the coupling pairs which admit polytope-duality extend to lattice duality of families $\mathcal{F}_{\Delta}$ and $\mathcal{F}_{\Delta^{\prime}}$ in the sense that the relation

$$
\left(\operatorname{Pic}_{\Delta}\right)_{U^{\oplus 3} \oplus E_{8}^{\oplus 2}}^{\perp} \simeq U \oplus \operatorname{Pic}_{\Delta^{\prime}}
$$

holds.

We give an answer as the main theorem of the article which is presented here:

Theorem 3.1 If a coupling pair admits polytope-duality with trivial toric contribution, then, the families of $K 3$ surfaces are lattice dual. Explicite Picard lattices of the families are given in Table 1.

In Sect. 2, we recall the Picard lattice and toric geometry. In Sect. 3, we give a proof of the main theorem. In the last and fourth section, we give a conclusion as the property of the Picard lattices of families that we have obtained.

\section{Preliminary}

A lattice is a finitely-generated $\mathbb{Z}$-module with a non-degenerate bilinear form. A $K 3$ surface is a smooth compact complex connected 2-dimensional algebraic variety with trivial canonical divisor and irregularity zero. It is known that the second cohomology group with $\mathbb{Z}$-coefficient of a $K 3$ surface $S$ admits a structure of a unimodular lattice of signature $(3,19)$, thus by a classification of lattices, the lattice is in fact isometric to the $K 3$ lattice $\Lambda_{K 3}:=U^{\oplus 3} \oplus E_{8}^{\oplus 2}$, where $U$ is the hyperbolic lattice of rank 2, and $E_{8}$ is the negative-definite, even unimodular lattice of rank 8 . By a standard exact sequence, one gets an inclusion map $c_{1}: H^{1}\left(S, \mathcal{O}_{S}^{*}\right) \rightarrow H^{2}(S, \mathbb{Z})$, which makes the Picard group $H^{1}\left(S, \mathcal{O}_{S}^{*}\right)$ to be a sublattice of $H^{2}(S, \mathbb{Z})$. We call the Picard group of $S$ with a lattice structure simply the Picard lattice of $S$.

We summarize toric geometry in Batyrev (1994) by also giving useful formulas extracted from Fulton (1997) and Oda (1978).

Let $M$ be a lattice of $\operatorname{rank} n$, and $N:=\operatorname{Hom}_{\mathbb{Z}}(M \mathbb{Z})$ be the dual lattice of $M$, with a natural pairing $\langle\rangle:, N \times M \rightarrow \mathbb{Z}$ with its $\mathbb{R}$-extension denoted by $\langle,\rangle_{\mathbb{R}}$. A convex hull of finite-number of points in $M \otimes \mathbb{R}$ is called a polytope, which admits the polar dual polytope $\Delta^{*}$ defined by

$$
\Delta^{*}:=\left\{y \in N \otimes \mathbb{R} \mid\langle y, x\rangle_{\mathbb{R}} \geq-1 \text { for all } x \in \Delta\right\} .
$$

A polytope $\Delta$ is integral if every vertex is in $M$. An integral polytope $\Delta$ which contains the only lattice point in its interior is reflexive if the polar dual polytope $\Delta^{*}$ is also an integral polytope. 
It is observed by Batyrev (1994) that an integral polytope $\Delta$ is reflexive if and only if the resulting projective toric variety $\mathbb{P}_{\Delta}$ is Fano, in other words, general hypersurfaces that are defined by global anticanonical sections of $\mathbb{P}_{\Delta}$ are birational to Calabi-Yau.

We only treat with 3-dimensional reflexive polytopes. We call a anticanonical section for hypersurfaces that are defined by global anticanonical sections of $\mathbb{P}_{\Delta}$ for short. In 3-dimensional case, it is derived by a study of Batyrev (1994), that moreover, singularities in $\mathbb{P}_{\Delta}$ and in general anticanonical sections $Z$ of $\mathbb{P}_{\Delta}$ can be simultaneously resolved by a toric resolution called a MPCP-desingularisation, which we denote by $\widetilde{\mathbb{P}_{\Delta}}$ and $\tilde{Z}$. The natural restriction map

$$
H^{1,1}\left(\widetilde{\mathbb{P}_{\Delta}}, \mathbb{Z}\right) \rightarrow H^{1,1}(\tilde{Z}, \mathbb{Z})
$$

is not necessarily subjective in general, and we denote by $L_{0}(\Delta)$ the rank of the cokernel of the map, which we call the toric contribution, which is known Kobayashi (2008) to be given by the formula

$$
L_{0}(\Delta)=\sum_{\Gamma} l(\Gamma) l\left(\Gamma^{*}\right)
$$

where the sum runs for all edges in $\Delta$.

Here we recall from Bruzzo and Grassi (2012) that generic anticanonical sections of the Fano threefold $\mathbb{P}_{\Delta}$ admit isometric Picard lattices. Thus, we define the Picard lattice of the family $\mathcal{F}_{\Delta}$ of $K 3$ surfaces in $\mathbb{P}_{\Delta}$ to be the Picard lattice of the minimal model of any generic anticanonical section of $\mathbb{P}_{\Delta}$, and denote it by $\mathrm{Pic}_{\Delta}$.

For a reflexive polytope $\Delta$, one can associate a fan $\Sigma^{\prime}$. By definition, lattice points of $\Delta^{*}$ are primitive vector of one-simplices of $\Sigma^{\prime}$, and it is clear that the toric varieties $\mathbb{P}_{\Delta}$ and $\mathbb{P}_{\Sigma^{\prime}}$ coincide. Any divisor $D$ of a generic hypersurface in $\mathbb{P}_{\Delta}$ is the closure of the torus orbit of a one-simplex $v$ in $\Sigma$, in particular, the divisors are called toric divisors. Let $F$ be the face in $\Delta$ that is the polar dual of $v$. Denote by $l(F)$ the number of lattice points in the interior of $F$. The self-intersection number of the divisor $D$ is given by the formula

$$
D^{2}=2 l(F)-2
$$

Denote by $\Delta^{(1)}$ the set of all edges in $\Delta$ and $l(\Gamma)$ be the number of lattice points in the interior of an edge $\Gamma \in \Delta^{(1)}$. The Picard number $\rho(\Delta)$ is given by

$$
\rho(\Delta)=\sum_{\Gamma \in \Delta^{(1)}} l(\Gamma)+\sum_{\text {vertices of } \Delta} 1+L_{0}(\Delta)-3,
$$

Let $e_{1}, e_{2}, e_{3}$ be a standard basis for $\mathbb{R}^{3}$. Suppose that the fan $\Sigma$ possesses $l$ onesimplices. The toric divisors $D_{1}, \ldots, D_{l}$ admit the linear relations

$$
\sum_{i=1}^{l}\left\langle v_{i}, e_{j}\right\rangle D_{i}=0 \quad j=1,2,3 .
$$


It is easily seen that the polytope $\Delta$ is of trivial toric contribution if and only if the corresponding fan $\Sigma^{\prime}$ is simplicial, that is, every triple of one-simplices form a $\mathbb{Z}$-basis of $\mathbb{R}^{3}$. Moreover, the restriction of linearly-independent toric divisors of $X=\widetilde{\mathbb{P}_{\Delta}}=\widetilde{\mathbb{P}_{\Sigma^{\prime}}}$ to the anticanonical divisor of $X$ form a basis of the Picard lattice Pic $_{\Delta^{\prime}}$ of the family $\mathcal{F}_{\Delta^{\prime}}$ if $\Delta^{\prime} \simeq \Delta^{*}$.

Denote by $M_{\left(a_{0}, a_{1}, a_{2}, a_{3}\right)}$ the lattice consisting of quadruple of integers $(i, j, k, l)$ satisfying an equation $a_{0} i+a_{1} j+a_{2} k+a_{3} l=0$ for a weight system $\left(a_{0}, a_{1}, a_{2}, a_{3} ; d\right)$. There is a one-to-one correspondence between elements in $M_{\left(a_{0}, a_{1}, a_{2}, a_{3}\right)}$ and (rational) monomials of degree $d$ by

$$
(i, j, k, l) \in M_{\left(a_{0}, a_{1}, a_{2}, a_{3}\right)} \leftrightarrow W^{i+1} X^{j+1} Y^{k+1} Z^{l+1},
$$

where $(W, X, Y, Z)$ is a coordinate system of the weighted projective space of weight $\left(a_{0}, a_{1}, a_{2}, a_{3}\right)$.

We denote by $L^{*}, A_{L}$, discr $L, l\left(A_{L}\right), \operatorname{sgn} L, q_{L}$, and rank $L$ the dual lattice $L^{*}:=\operatorname{Hom}_{\mathbb{Z}}(L, \mathbb{Z})$, the discriminant group $L / L^{*}$, the discriminant, the minimal number of generators of $A_{L}$, the signature, the discriminant form, and the rank of a lattice $L$. It is a standard arithmetic property that if $\operatorname{rank} L$ is strictly larger than 5 , then, there eists an element representing 0 , and if $\operatorname{rank} L$ is strictly larger than 12 , then, the hyperbolic lattice $U$ is a sublattice of $L$. We also recall standard properties of lattices from Nikulin (1980) and Nishiyama (1996). A sublattice $S$ of a lattice $\Lambda$ is called primitive if the quotient lattice $\Lambda / S$ is torsion-free.

Corollary 2.1 (Corollary 1.6.2 Nikulin (1980)) Let $S$ and $T$ be primitive sublattices of the $K 3$ lattice $\Lambda_{K 3}$. The lattices $S$ and $T$ are orthogonal in $\Lambda_{K 3}$ if and only if $q_{S} \simeq-q_{T}$ holds.

Corollary 2.2 (Corollary 1.12.3 Nikulin (1980)) Let $S$ be a sublattice with signature $\left(t_{+}, t_{-}\right)$of an even unimodular lattice $\Lambda$ with signature $\left(l_{+}, l_{-}\right)$. The lattice $S$ is a primitive sublattice of $\Lambda$ if and only if the following three conditions are satisfied.

(1) $l_{+}-l_{-} \equiv 0 \bmod 8$,

(2) $l_{-}-t_{-} \geq 0$ and $l_{+}-t_{+} \geq 0$, and

(3) $\operatorname{rank} \Lambda-\operatorname{rank} S>l\left(A_{S}\right)$.

Remark 1 Note that the $K 3$ lattice $\Lambda_{K 3}$ is an even unimodular lattice of signature $\left(l_{+}, l_{-}\right)=(3,19)$. Thus, $l_{+}-l_{-}=3-19=-16 \equiv 0 \bmod 8$, and in order to show a lattice $S$ to be a primitive sublattice of $\Lambda_{K 3}$, it suffices to verify the second and third conditions of Corollary 2.2.

Lemma 2.1 (Lemma 4.3 Nishiyama (1996)) There exist primitive embeddings of $A_{1}$ and $A_{2}$ into $E_{8}$ with orthogonal complements being $E_{7}$ and $E_{6}$, respectively. We follow the notation of lattices in Bourbaki (1968). 


\section{Main results}

Lemma 3.1 The polytope-dual pairs among Nos. 11-14, Nos. 15-18, Nos.35-37, Nos.38 and 40, Nos.41-43, Nos.48-49 are respectively isomorphic to the following polytopes in Table 1.

Proof The assertion follows from the proof of Mase (2021).

Lemma 3.2 If a coupling pair is in Table 1, the toric contribution is trivial.

Proof The assertion follows by case-by-case computation using formula (1) for all polytopes obtained in Mase (2021).

Theorem 3.1 If a coupling pair admits polytope-duality with trivial toric contribution, then, the families of $K 3$ surfaces are lattice dual. Explicite Picard lattices of the families are given in Table 1.

Remark 2 We present the following data in Table 1. The number(s) in the first column are given in Mase (2021). The second and fifth columns are vertices of polytopes of $\Delta^{\prime}$ and $\Delta$ obtained by Mase (2021), and the sets in the same line are polytope-dual. In the third and fourth columns are the Picard lattice of the family $\mathcal{F}_{\Delta^{\prime}}$, resp. $\mathcal{F}_{\Delta}$, the pair of the rank and the signature of lattices, and the weight systems that are coupling. The lattices $\tilde{L}$ and $\tilde{L}^{\prime}$ are explained in the proof. Other lattices follow notation of Bourbaki (1968).

Proof Take reflexive polytopes $\Delta$ and $\Delta^{\prime}$ as in 1 . We explicitly calculate the Picard lattices of the families $\mathcal{F}_{\Delta}$ and $\mathcal{F}_{\Delta^{\prime}}$. Denote by $\Sigma$, respectively $\Sigma^{\prime}$ the fan associated to polytope $\Delta^{\prime}$, respectively $\Delta$. Since the relation $\Delta^{*} \simeq \Delta^{\prime}$ holds, lattice points of $\Delta^{\prime}$, respectively of $\Delta$ are none other than primitive vectors of one-simplices of $\Sigma$, respectively $\Sigma^{\prime}$.

\subsection{Nos. 11, 12, 13, and 14}

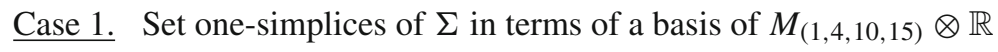

$$
\begin{aligned}
& (-1,4,0,-1),(-1,-1,2,-1),(-1,-1,-1,1) \text { : } \\
& v_{1}=(1,0,0), \quad v_{2}=(0,1,0), \quad v_{3}=(0,0,1), \\
& v_{4}=(0,-2,-3), \quad v_{5}=(-6,-8,-15), \quad v_{6}=(-3,-4,-7) \text {, } \\
& v_{7}=(0,-1,-1), \quad v_{8}=(-2,-2,-5), \quad v_{9}=(-4,-5,-10) \text {, } \\
& v_{10}=(-5,-7,-13), v_{11}=(-4,-6,-11), v_{12}=(-3,-5,-9) \text {, } \\
& v_{13}=(-2,-4,-7), \quad v_{14}=(-1,-3,-5) \text {, }
\end{aligned}
$$

and let $\tilde{D}_{i}$ be the toric divisor determined by the lattice point $v_{i}$ for $i=1, \ldots, 14$, and $D_{i}:=\left.\tilde{D}_{i}\right|_{-K_{X}}$ with $X:=\widetilde{\mathbb{P}_{\Sigma}}$. It can be easily seen by formulas (3) and (2) that

$$
\rho_{\Delta}=14-3=11, \quad D_{1}^{2}=0, D_{2}^{2}=2, D_{3}^{2}=8, D_{4}^{2}=\cdots=D_{14}^{2}=-2 .
$$




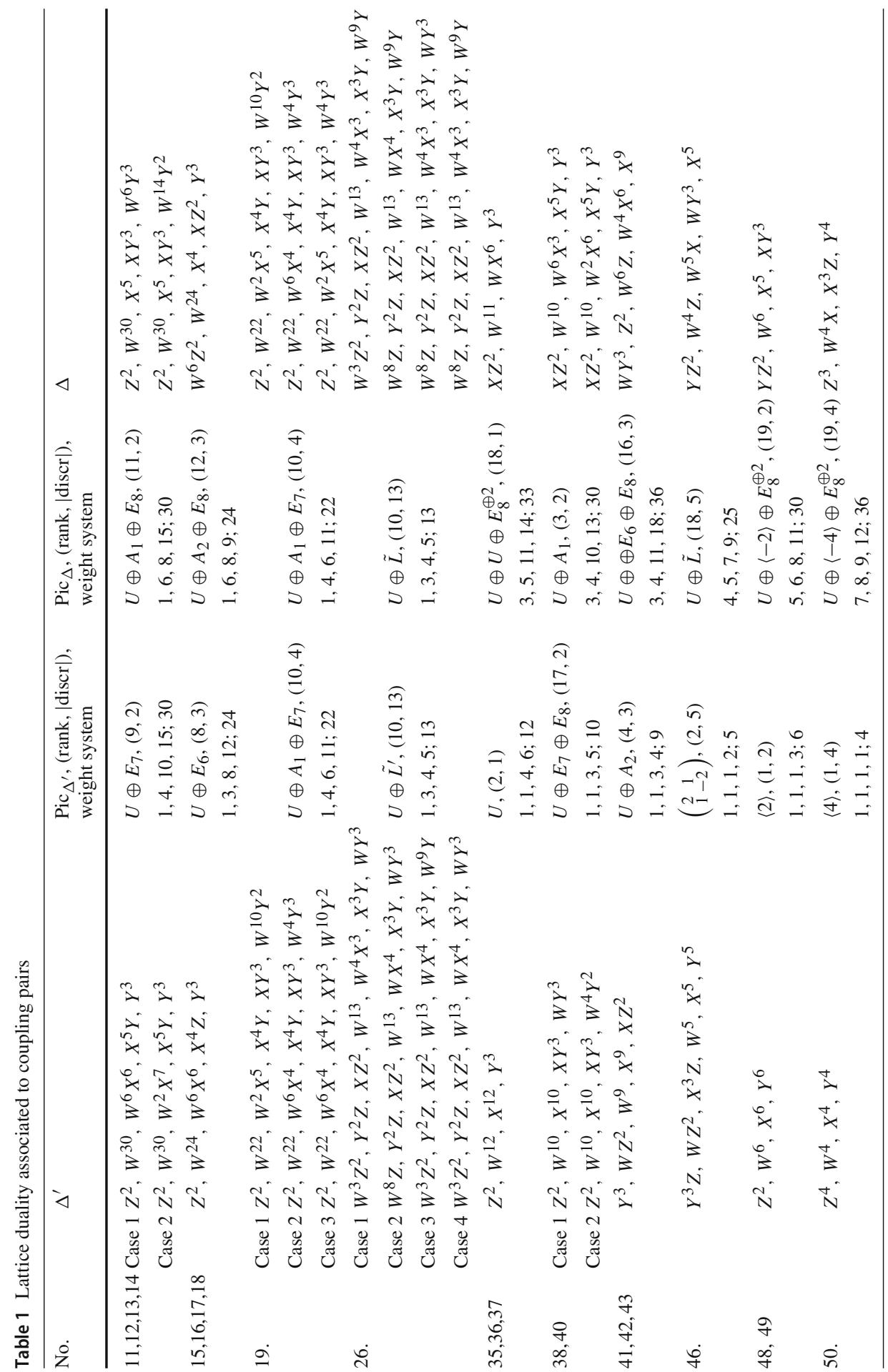


Let $L$ be a lattice generated by divisors $\left\{D_{i}\right\}_{i=1}^{14}$. By solving the Eq. (4), one sees that $\left\{D_{1}, D_{2}, D_{8}, D_{4}, D_{7}, D_{14}, D_{13}, D_{12}, D_{11}, D_{10}, D_{5}\right\}$ form a basis for $L$. By taking a new basis

$$
\left\{\begin{array}{l}
D_{1}, D_{1}+D_{4},-D_{1}+D_{7}, D_{8} \\
2 D_{1}-D_{2}+2 D_{4}+D_{7}-D_{8}+D_{14} \\
D_{13},-D_{1}+D_{14}, D_{12}, D_{11}, D_{10}, D_{5}
\end{array}\right\}
$$

one sees that the lattice $L$ is isometric to $U \oplus A_{1} \oplus E_{8}$, which is hyperbolic and a primitive sublattice of the $K 3$ lattice. Therefore, $\operatorname{Pic}_{\Delta} \simeq U \oplus A_{1} \oplus E_{8}$.

Set one-simplices of $\Sigma^{\prime}$ in terms of a basis of $M_{(1,6,8,15)} \otimes \mathbb{R}$

$$
\begin{aligned}
& (-6,1,0,0),(-8,0,1,0),(-15,0,0,1) \text { : } \\
& m_{1}=(4,-1,-1), \quad m_{2}=(0,2,-1), \quad m_{3}=(-1,-1,1) \text {, } \\
& m_{4}=(-1,2,-1), \quad m_{5}=(-1,-1,-1), m_{6}=(3,-1,-1) \text {, } \\
& m_{7}=(2,-1,-1), \quad m_{8}=(1,-1,-1), \quad m_{9}=(0,-1,-1) \text {, } \\
& m_{10}=(-1,1,-1), m_{11}=(-1,0,-1), m_{12}=(-1,-1,0) \text {, }
\end{aligned}
$$

and let $\tilde{D}_{i}^{\prime}$ be the toric divisor determined by the lattice point $m_{i}$ for $i=1, \ldots, 12$, and $D_{i}^{\prime}:=\left.\tilde{D}_{i}^{\prime}\right|_{-K_{X}}$ with $X:=\widetilde{\mathbb{P}_{\Sigma^{\prime}}}$. It can be easily seen by formulas (3) and (2) that

$$
\rho_{\Delta^{\prime}}=12-3=9, \quad D_{1}^{\prime 2}=0, D_{2}^{\prime 2}=2, D_{3}^{\prime 2}=10, D_{4}^{\prime 2}=\cdots=D_{12}^{\prime 2}=-2 .
$$

Let $L$ be a lattice generated by divisors $\left\{D_{i}^{\prime}\right\}_{i=1}^{12}$. By solving the Eq. (4), one sees that $\left\{D_{1}^{\prime}, D_{2}^{\prime}, D_{4}^{\prime}, D_{10}^{\prime}, D_{11}^{\prime}, D_{5}^{\prime}, D_{6}^{\prime}, D_{7}^{\prime}, D_{8}^{\prime}\right\}$ form a basis for $L$. By taking a new basis

$$
\left\{\begin{array}{l}
D_{1}^{\prime}, D_{1}^{\prime}+D_{6}^{\prime}, D_{4}^{\prime}, D_{10}^{\prime}, D_{11}^{\prime}, D_{5}^{\prime}, \\
-3 D_{1}^{\prime}+D_{2}^{\prime}+D_{4}^{\prime}-2 D_{6}^{\prime}-D_{7}^{\prime}-D_{8}^{\prime},-D_{1}^{\prime}+D_{7}^{\prime}+D_{8}^{\prime}, D_{1}^{\prime}-D_{7}^{\prime}
\end{array}\right\},
$$

one sees that the lattice $L^{\prime}$ is isometric to $U \oplus E_{7}$, which is hyperbolic and a primitive sublattice of the $K 3$ lattice. Therefore, $\operatorname{Pic}_{\Delta^{\prime}} \simeq U \oplus E_{7}$.

$\underline{\text { Case } 2}$ Set one-simplices of $\Sigma$ in terms of a basis of $M_{(1,4,10,15)} \otimes \mathbb{R}$

$$
\begin{aligned}
& (-4,1,0,0),(-10,0,1,0),(-15,0,0,1): \\
& v_{1}=(-1,-1,1), \quad v_{2}=(-1,-1,-1), v_{3}=(6,-1,-1) \text {, } \\
& v_{4}=(4,0,-1), \quad v_{5}=(-1,2,-1), \quad v_{6}=(-1,-1,0) \text {, } \\
& v_{7}=(0,-1,-1), \quad v_{8}=(1,-1,-1), \quad v_{9}=(2,-1,-1) \text {, } \\
& v_{10}=(3,-1,-1), v_{11}=(4,-1,-1), \quad v_{12}=(5,-1,-1) \text {, } \\
& v_{13}=(-1,0,-1), v_{14}=(-1,1,-1) \text {, }
\end{aligned}
$$

and let $\tilde{D}_{i}$ be the toric divisor determined by the lattice point $v_{i}$ for $i=1, \ldots, 14$, and $D_{i}:=\left.\tilde{D}_{i}\right|_{-K_{X}}$ with $X:=\widetilde{\mathbb{P}_{\Sigma}}$. It can be easily seen by formulas (3) and (2) that

$$
\begin{aligned}
& \rho_{\Delta}=14-3=11, D_{1}^{2}=8, D_{2}^{2}=D_{3}^{2}=D_{4}^{2}=-2, D_{5}^{2}=2 \text {, } \\
& D_{6}^{2}=\cdots=D_{14}^{2}=-2 \text {. }
\end{aligned}
$$


Let $L$ be a lattice generated by divisors $\left\{D_{i}\right\}_{i=1}^{14}$. By solving the Eq. (4), one sees that $\left\{D_{4}, D_{3}, D_{5}, D_{14}, D_{13}, D_{2}, D_{12}, D_{11}, D_{10}, D_{9}, D_{8}\right\}$ form a basis for $L$. By taking a new basis

$$
\left\{\begin{array}{l}
-D_{3}+D_{5}-D_{12}, D_{3}+D_{4}, D_{4}, D_{13}, \\
-D_{2}-D_{13}, D_{2}-D_{3}-D_{4}+D_{13}+D_{14}, \\
2 D_{3}+D_{4}-D_{5}+D_{11}+2 D_{12}-D_{13}-D_{14} \\
D_{3}+D_{4}+D_{11}, D_{10}, D_{9}, D_{8}
\end{array}\right\},
$$

one sees that the lattice $L$ is isometric to $U \oplus A_{1} \oplus E_{8}$, which is hyperbolic and a primitive sublattice of the $K 3$ lattice. Therefore, $\operatorname{Pic}_{\Delta} \simeq U \oplus A_{1} \oplus E_{8}$.

Set one-simplices of $\Sigma^{\prime}$ in terms of a basis of $M_{(1,6,8,15)} \otimes \mathbb{R}$

$$
\begin{aligned}
& (-6,1,0,0),(-8,0,1,0),(-15,0,0,1) \text { : } \\
& m_{1}=(-1,-1,1), \quad m_{2}=(-1,-1,-1), m_{3}=(4,-1,-1) \text {, } \\
& m_{4}=(0,2,-1), \quad m_{5}=(-1,1,-1), \quad m_{6}=(-1,-1,0) \text {, } \\
& m_{7}=(0,-1,-1), \quad m_{8}=(1,-1,-1), \quad m_{9}=(2,-1,-1), \\
& m_{10}=(3,-1,-1), m_{11}=(-1,0,-1), m_{12}=(-1,0,0) \text {, }
\end{aligned}
$$

and let $\tilde{D}_{i}^{\prime}$ be the toric divisor determined by the lattice point $m_{i}$ for $i=1, \ldots, 12$, and $D_{i}^{\prime}:=\tilde{D}_{i}^{\prime} \mid-K_{X}$ with $X:=\widetilde{\mathbb{P}_{\Sigma^{\prime}}}$. It can be easily seen by formulas (3) and (2) that

$$
\begin{aligned}
& \rho_{\Delta^{\prime}}=12-3=9, D_{1}^{\prime 2}=10, D_{2}^{\prime 2}=-2, D_{3}^{\prime 2}=0, D_{4}^{\prime 2}=4 \text {, } \\
& D_{5}^{\prime 2}=\cdots=D_{12}^{\prime 2}=-2 \text {. }
\end{aligned}
$$

Let $L^{\prime}$ be a lattice generated by divisors $\left\{D_{i}^{\prime}\right\}_{i=1}^{12}$. By solving the Eq. (4), one sees that $\left\{D_{3}^{\prime}, D_{10}^{\prime}, D_{8}^{\prime}, D_{6}^{\prime}, D_{7}^{\prime}, D_{2}^{\prime}, D_{11}^{\prime}, D_{5}^{\prime}, D_{12}^{\prime}\right\}$ form a basis for $L^{\prime}$, with respect to which the intersection matrix of $L^{\prime}$ is $U \oplus E_{7}$, which is hyperbolic and a primitive sublattice of the $K 3$ lattice. Therefore, Pic $_{\Delta^{\prime}} \simeq U \oplus E_{7}$.

It is well-known that lattices $U \oplus A_{1} \oplus E_{8}$ and $U \oplus E_{7}$ are primitive sublattices of the $K 3$ lattice $\Lambda_{K 3}$. Moreover, by Lemma 2.1, the relation $\left(\mathrm{Pic}_{\Delta}\right)_{\Lambda_{K 3}}^{\perp} \simeq U_{U^{\oplus 3}}^{\perp} \oplus$ $\left(A_{1}\right) \stackrel{\perp}{E_{8}} \oplus\left(E_{8}\right) \stackrel{\perp}{E_{8}}=U^{\oplus 2} \oplus E_{7} \simeq U \oplus \mathrm{Pic}_{\Delta^{\prime}}$ holds.

\subsection{Nos. 15, 16, 17, and 18}

Set one-simplices of $\Sigma$ in terms of a basis of $M_{(1,6,8,9)} \otimes \mathbb{R}$

$$
\begin{aligned}
& (-6,1,0,0),(-8,0,1,0),(-9,0,0,1) \text { : } \\
& v_{1}=(-1,2,-1), \quad v_{2}=(-1,-1,-1), v_{3}=(5,-1,-1) \text {, } \\
& v_{4}=(3,-1,0), \quad v_{5}=(-1,-1,1), \quad v_{6}=(-1,1,-1) \text {, } \\
& v_{7}=(-1,0,-1), \quad v_{8}=(0,-1,-1), \quad v_{9}=(1,-1,-1) \text {, } \\
& v_{10}=(2,-1,-1), v_{11}=(3,-1,-1), \quad v_{12}=(4,-1,-1) \text {, } \\
& v_{13}=(-1,-1,0), v_{14}=(3,0,-1), \quad v_{15}=(1,1,-1) \text {, }
\end{aligned}
$$


and let $\tilde{D}_{i}$ be the toric divisor determined by the lattice point $v_{i}$ for $i=1, \ldots, 15$, and $D_{i}:=\left.\tilde{D}_{i}\right|_{-K_{X}}$ with $X:=\widetilde{\mathbb{P}_{\Sigma}}$. It can be easily seen by formulas (3) and (2) that

$$
\begin{aligned}
\rho_{\Delta}=15-3=12, D_{1}^{2} & =2, D_{2}^{2}=D_{3}^{2}=-2 \\
D_{4}^{2} & =0, D_{5}^{2}=4, D_{6}^{2}=\cdots=D_{15}^{2}=-2 .
\end{aligned}
$$

Let $L$ be a lattice generated by divisors $\left\{D_{i}\right\}_{i=1}^{15}$. By solving the Eq. (4), one sees that $\left\{D_{4}, D_{5}, D_{13}, D_{2}, D_{7}, D_{3}, D_{14}, D_{15}, D_{12}, D_{11}, D_{10}, D_{9}\right\}$ form a basis for $L$. By taking a new basis

$$
\left\{\begin{array}{l}
D_{4}, D_{3}+D_{4}, D_{4}-D_{14}-D_{15},-D_{4}+D_{14},-D_{7}, \\
-D_{13},-D_{4}+D_{11}+D_{12}, \\
3 D_{3}+2 D_{4}-D_{5}+D_{10}+2 D_{11}+2 D_{12}+2 D_{14}+D_{15}, \\
-D_{2},-D_{9}-D_{10}-D_{11}, D_{10}, D_{9}
\end{array}\right\}
$$

one sees that the lattice $L$ is isometric to $U \oplus A_{2} \oplus E_{8}$, which is hyperbolic and a primitive sublattice of the $K 3$ lattice. Therefore, $\operatorname{Pic}_{\Delta} \simeq U \oplus A_{2} \oplus E_{8}$.

Set one-simplices of $\Sigma^{\prime}$ in terms of a basis of $M_{(1,3,8,12)} \otimes \mathbb{R}$

$$
\begin{aligned}
& (-3,1,0,0),(-8,0,1,0),(-12,0,0,1) \text { : } \\
& m_{1}=(-1,2,-1), \quad m_{2}=(-1,-1,-1), m_{3}=(3,-1,-1) \text {, } \\
& m_{4}=(0,-1,1), \quad m_{5}=(-1,-1,1), \quad m_{6}=(-1,1,-1) \text {, } \\
& m_{7}=(-1,0,-1), \quad m_{8}=(0,-1,1), \quad m_{9}=(1,-1,-1) \text {, } \\
& m_{10}=(2,-1,-1), m_{11}=(-1,-1,0) \text {, }
\end{aligned}
$$

and let $\tilde{D}_{i}^{\prime}$ be the toric divisor determined by the lattice point $m_{i}$ for $i=1, \ldots, 11$, and $D_{i}^{\prime}:=\left.\tilde{D}_{i}^{\prime}\right|_{-K_{X}}$ with $X:=\widetilde{\mathbb{P}_{\Sigma^{\prime}}}$. It can be easily seen by formulas (3) and (2) that

$$
\begin{aligned}
& \rho_{\Delta^{\prime}}=11-3=8, D_{1}^{\prime 2}=4, D_{2}^{\prime 2}=-2, D_{3}^{\prime 2}=0 \\
& D_{4}^{\prime 2}=6, D_{5}^{\prime 2}=-2, D_{6}^{\prime 2}=\cdots=D_{11}^{\prime 2}=-2 .
\end{aligned}
$$

Let $L^{\prime}$ be a lattice generated by divisors $\left\{D_{i}^{\prime}\right\}_{i=1}^{11}$. By solving the Eq. (4), one sees that $\left\{D_{4}^{\prime}, D_{3}^{\prime}, D_{10}^{\prime}, D_{9}^{\prime}, D_{5}^{\prime}, D_{11}^{\prime}, D_{2}^{\prime}, D_{7}^{\prime}\right\}$ form a basis for $L^{\prime}$. By taking a new basis

$$
\left\{\begin{array}{l}
D_{4}^{\prime}-D_{3}^{\prime}, 2 D_{3}^{\prime}-D_{5}^{\prime}+D_{9}^{\prime}+2 D_{10}^{\prime},-D_{3}^{\prime}+D_{5}^{\prime}, \\
3 D_{3}^{\prime}-2 D_{5}^{\prime}+D_{9}^{\prime}+3 D_{10}^{\prime}-D_{11}^{\prime} \\
D_{9}^{\prime},-D^{\prime}+D_{4}^{\prime}+D_{11}^{\prime} \\
D_{2}^{\prime}-3 D_{3}^{\prime}+2 D_{5}^{\prime}-2 D_{9}^{\prime}-3 D_{10}^{\prime}+D_{11}^{\prime}, D_{7}^{\prime}
\end{array}\right\}
$$

one sees that the lattice $L^{\prime}$ is isometric to $U \oplus E_{6}$, which is hyperbolic and a primitive sublattice of the $K 3$ lattice. Therefore, $\operatorname{Pic}_{\Delta^{\prime}} \simeq U \oplus E_{6}$.

It is well-known that lattices $U \oplus A_{2} \oplus E_{8}$ and $U \oplus E_{6}$ are primitive sublattices of the $K 3$ lattice $\Lambda_{K 3}$. Moreover, by Lemma 2.1, the relation $\left(\operatorname{Pic}_{\Delta}\right)_{\Lambda_{K 3}}^{\perp} \simeq U_{U^{\oplus 3}}^{\perp} \oplus$ $\left(A_{2}\right)_{E_{8}}^{\perp} \oplus\left(E_{8}\right) \frac{\perp}{E_{8}}=U^{\oplus 2} \oplus E_{6} \simeq U \oplus \mathrm{Pic}_{\Delta^{\prime}}$ holds. 


\subsection{No. 19}

In all cases, we set one-simplices in fans in terms of a basis of $M_{(1,4,6,11)} \otimes \mathbb{R}$

$$
(-4,1,0,0),(-6,0,1,0),(-11,0,0,1)
$$

Case 1 We have $\Delta \simeq \Delta^{\prime}$.

Set one-simplices of $\Sigma^{\prime}$ as follows:

$$
\begin{array}{lll}
m_{1}=(-1,-1,1), & m_{2}=(-1,-1,-1), & m_{3}=(4,-1,-1), \\
m_{4}=(3,0,-1), & m_{5}=(0,2,-1), & m_{6}=(-1,1,-1), \\
m_{7}=(-1,-1,0), & m_{8}=(0,-1,-1), & m_{9}=(1,-1,-1), \\
m_{10}=(2,-1,-1), & m_{11}=(3,-1,-1), & m_{12}=(-1,0,0), \\
m_{13}=(-1,0,-1), &
\end{array}
$$

and let $\tilde{D}_{i}^{\prime}$ be the toric divisor determined by the lattice point $m_{i}$ for $i=1, \ldots, 13$, and $D_{i}^{\prime}:=\tilde{D}_{i}^{\prime} \mid-K_{X}$ with $X:=\widetilde{\mathbb{P}_{\Sigma^{\prime}}}$. It can be easily seen by formulas (3) and (2) that

$$
\begin{aligned}
& \rho_{\Delta^{\prime}}=13-3=10, D_{1}^{\prime 2}=8, D_{2}^{\prime 2}=D_{3}^{\prime 2}=D_{4}^{\prime 2}=-2, D_{5}^{\prime 2}=2 \text {, } \\
& D_{6}^{\prime 2}=\cdots=D_{13}^{\prime 2}=-2 \text {. }
\end{aligned}
$$

Let $L^{\prime}$ be a lattice generated by divisors $\left\{D_{i}^{\prime}\right\}_{i=1}^{13}$. By solving the Eq. (4), one sees that $\left\{D_{4}^{\prime}, D_{5}^{\prime}, D_{3}^{\prime}, D_{11}^{\prime}, D_{10}^{\prime}, D_{9}^{\prime}, D_{6}^{\prime}, D_{12}^{\prime}, D_{13}^{\prime}, D_{2}^{\prime}\right\}$ form a basis for $L^{\prime}$. By taking a new basis

$$
\left\{\begin{array}{l}
D_{4}^{\prime},-D_{3}^{\prime}+D_{5}^{\prime}, D_{3}^{\prime}+D_{4}^{\prime}+D_{11}^{\prime}, \\
-3 D_{3}^{\prime}-2 D_{4}^{\prime}+D_{5}^{\prime}+D_{6}^{\prime}-D_{9}^{\prime}-2 D_{10}^{\prime}-3 D_{11}^{\prime}+D_{13}^{\prime}, \\
D_{3}^{\prime}-D_{5}^{\prime}+D_{10}^{\prime}, \\
3 D_{3}^{\prime}+2 D_{4}^{\prime}-D_{5}^{\prime}-2 D_{6}^{\prime}+D_{10}^{\prime}+2 D_{11}^{\prime}-D_{12}^{\prime}-D_{13}^{\prime}, \\
5 D_{3}^{\prime}+3 D_{4}^{\prime}-2 D_{5}^{\prime}-2 D_{6}^{\prime}+2 D_{9}^{\prime}+3 D_{10}^{\prime}+4 D_{11}^{\prime}-D_{12}^{\prime}-D_{13}^{\prime}, \\
D_{2}^{\prime}-5 D_{3}^{\prime}-3 D_{4}^{\prime}+2 D_{5}^{\prime}+2 D_{6}^{\prime}-D_{9}^{\prime}-3 D_{10}^{\prime}-4 D_{11}^{\prime}+D_{12}^{\prime}+D_{13}^{\prime}, \\
D_{12}^{\prime}, \\
-D_{2}^{\prime}+3 D_{3}^{\prime}+2 D_{4}^{\prime}-D_{5}^{\prime}-D_{6}^{\prime}+D_{9}^{\prime}+2 D_{10}^{\prime}+3 D_{11}^{\prime}-D_{12}^{\prime}-D_{13}^{\prime}
\end{array}\right\},
$$

one sees that the lattice $L^{\prime}$ is isometric to $U \oplus A_{1} \oplus E_{7}$, which is hyperbolic and a primitive sublattice of the $K 3$ lattice. Therefore, $\mathrm{Pic}_{\Delta^{\prime}} \simeq U \oplus A_{1} \oplus E_{7}$. By similar computation, one has $\mathrm{Pic}_{\Delta} \simeq U \oplus A_{1} \oplus E_{7}$.

Case 2 We have $\Delta \simeq \Delta^{\prime}$.

Set one-simplices of $\Sigma^{\prime}$ as follows:

$$
\begin{array}{lll}
m_{1}=(-1,-1,1), & m_{2}=(-1,-1,-1), & m_{3}=(3,-1,-1), \\
m_{4}=(3,0,-1), & m_{5}=(0,2,-1), & m_{6}=(-1,2,-1), \\
m_{7}=(-1,-1,0), & m_{8}=(0,-1,-1), & m_{9}=(1,-1,-1), \\
m_{10}=(2,-1,-1), & m_{11}=(1,-1,0), & m_{12}=(-1,0,-1), \\
m_{13}=(-1,1,-1), &
\end{array}
$$


and let $\tilde{D}_{i}^{\prime}$ be the toric divisor determined by the lattice point $m_{i}$ for $i=1, \ldots, 13$, and $D_{i}^{\prime}:=\left.\tilde{D}_{i}^{\prime}\right|_{-K_{X}}$ with $X:=\widetilde{\mathbb{P}_{\Sigma^{\prime}}}$. It can be easily seen by formulas (3) and (2) that

$$
\begin{aligned}
& \rho_{\Delta^{\prime}}=13-3=10, D_{1}^{\prime 2}=8, D_{2}^{\prime 2}=D_{3}^{\prime 2}=-2, D_{4}^{\prime 2}=D_{5}^{\prime 2}=0 \text {, } \\
& D_{6}^{\prime 2}=\cdots=D_{13}^{\prime 2}=-2 \text {. }
\end{aligned}
$$

Let $L^{\prime}$ be a lattice generated by divisors $\left\{D_{i}^{\prime}\right\}_{i=1}^{13}$. By solving the Eq. (4), one sees that $\left\{D_{4}^{\prime}, D_{3}^{\prime}, D_{11}^{\prime}, D_{9}^{\prime}, D_{8}^{\prime}, D_{2}^{\prime}, D_{7}^{\prime}, D_{12}^{\prime}, D_{13}^{\prime}, D_{6}^{\prime}\right\}$ form a basis for $L^{\prime}$. By taking a new basis

$$
\left\{D_{4}^{\prime}, D_{3}^{\prime}+D_{4}^{\prime}, D_{11}^{\prime}-D_{4}^{\prime}, D_{9}^{\prime}, D_{8}^{\prime}, D_{2}^{\prime}, D_{7}^{\prime}, D_{12}^{\prime}, D_{13}^{\prime}, D_{6}^{\prime}\right\}
$$

one sees that the lattice $L^{\prime}$ is isometric to $U \oplus A_{1} \oplus E_{7}$, which is hyperbolic and a primitive sublattice of the $K 3$ lattice. Therefore, $\operatorname{Pic}_{\Delta^{\prime}} \simeq U \oplus A_{1} \oplus E_{7}$. By similar computation, one has $\mathrm{Pic}_{\Delta^{\prime}} \simeq U \oplus A_{1} \oplus E_{7}$.

$\underline{\text { Case } 3}$ Set one-simplices of $\Sigma$ as follows:

$$
\begin{array}{lll}
v_{1}=(-1,-1,1), & v_{2}=(-1,-1,-1), & v_{3}=(3,-1,-1), \\
v_{4}=(3,0,-1), & v_{5}=(0,2,-1), & v_{6}=(-1,1,-1), \\
v_{7}=(-1,-1,0), & v_{8}=(0,-1,-1), & v_{9}=(1,-1,-1), \\
v_{10}=(2,-1,-1), & v_{11}=(1,-1,0), & v_{12}=(-1,0,-1), \\
v_{13}=(-1,0,0), &
\end{array}
$$

and let $\tilde{D}_{i}$ be the toric divisor determined by the lattice point $v_{i}$ for $i=1, \ldots, 13$, and $D_{i}:=\left.\tilde{D}_{i}\right|_{-K_{X}}$ with $X=\widetilde{\mathbb{P}_{\Sigma}}$. It can be easily seen by formulas (3) and (2) that

$$
\begin{gathered}
\rho_{\Delta}=13-3=10, D_{1}^{2}=8, D_{2}^{2}=D_{3}^{2}=-2, D_{4}^{2}=0, D_{5}^{2}=2, \\
D_{6}^{2}=\cdots=D_{13}^{2}=-2 .
\end{gathered}
$$

Let $L$ be a lattice generated by divisors $\left\{D_{i}\right\}_{i=1}^{13}$. By solving the Eq. (4), one sees that $\left\{D_{4}, D_{3}, D_{11}, D_{9}, D_{8}, D_{2}, D_{7}, D_{12}, D_{6}, D_{13}\right\}$ form a basis for $L$. By taking a new basis

$$
\left\{D_{4}, D_{3}+D_{4}, D_{11}-D_{4}, D_{9}, D_{8}, D_{2}, D_{7}, D_{12}, D_{6}, D_{13}\right\}
$$

one sees that the lattice $L$ is isometric to $U \oplus A_{1} \oplus E_{7}$, which is hyperbolic and a primitive sublattice of the $K 3$ lattice. Therefore, $\operatorname{Pic}_{\Delta} \simeq U \oplus A_{1} \oplus E_{7}$.

Set one-simplices of $\Sigma^{\prime}$ as follows:

$$
\begin{array}{lll}
m_{1}=(-1,-1,1), & m_{2}=(-1,-1,-1), & m_{3}=(4,-1,-1), \\
m_{4}=(3,0,-1), & m_{5}=(0,2,-1), & m_{6}=(-1,2,-1), \\
m_{7}=(-1,-1,0), & m_{8}=(0,-1,-1), & m_{9}=(1,-1,-1), \\
m_{10}=(2,-1,-1), & m_{11}=(3,-1,-1), & m_{12}=(-1,0,-1), \\
m_{13}=(-1,1,-1), &
\end{array}
$$


and let $\tilde{D}_{i}^{\prime}$ be the toric divisor determined by the lattice point $m_{i}$ for $i=1, \ldots, 13$, and $D_{i}^{\prime}:=\left.\tilde{D}_{i}^{\prime}\right|_{-K_{X}}$ with $X:=\widetilde{\mathbb{P}_{\Sigma^{\prime}}}$. It can be easily seen by formulas (3) and (2) that

$$
\begin{aligned}
& \rho_{\Delta^{\prime}}=13-3=10, D_{1}^{\prime 2}=8, D_{2}^{\prime 2}=D_{3}^{\prime 2}=D_{4}^{\prime 2}=-2, D_{5}^{\prime 2}=0 \text {, } \\
& D_{6}^{\prime 2}=\cdots=D_{13}^{\prime 2}=-2 \text {. }
\end{aligned}
$$

Let $L^{\prime}$ be a lattice generated by divisors $\left\{D_{i}^{\prime}\right\}_{i=1}^{13}$. By solving the Eq. (4), one sees that $\left\{D_{4}^{\prime}, D_{3}^{\prime}, D_{11}^{\prime}, D_{9}^{\prime}, D_{8}^{\prime}, D_{2}^{\prime}, D_{7}^{\prime}, D_{12}^{\prime}, D_{13}^{\prime}, D_{6}^{\prime}\right\}$ form a basis for $L^{\prime}$. By taking a new basis

$$
\left\{D_{3}^{\prime}+D_{4}^{\prime}+D_{11}^{\prime}, D_{3}^{\prime}+D_{4}^{\prime}, D_{4}^{\prime}, D_{9}^{\prime}, D_{8}^{\prime}, D_{2}^{\prime}, D_{7}^{\prime}, D_{12}^{\prime}, D_{13}^{\prime}, D_{6}^{\prime}\right\},
$$

one sees that the lattice $L^{\prime}$ is isometric to $U \oplus A_{1} \oplus E_{7}$, which is hyperbolic and a primitive sublattice of the $K 3$ lattice. Thus, $\operatorname{Pic}_{\Delta^{\prime}} \simeq U \oplus A_{1} \oplus E_{7}$.

It is well-known that the lattice $U \oplus A_{1} \oplus E_{7}$ is a primitive sublattice of the $K 3$ lattice $\Lambda_{K 3}$. Moreover, by Lemma 2.1, the relation $\left(\mathrm{Pic}_{\Delta}\right)_{\Lambda_{K 3}}^{\perp} \simeq U_{U^{\oplus 3}}^{\perp} \oplus\left(A_{1}\right)_{E_{8}}^{\perp} \oplus\left(E_{7}\right)_{E_{8}}^{\perp}=$ $U^{\oplus 2} \oplus E_{7} \oplus A_{1} \simeq U \oplus \mathrm{Pic}_{\Delta^{\prime}}$ holds.

\subsection{No. 26}

In all cases, we set one-simplices of fans in terms of a basis of $M_{(1,3,4,5)} \otimes \mathbb{R}$

$$
(-3,1,0,0),(-4,0,1,0),(-5,0,0,1)
$$

Lemma 3.3 If lattices $L$ and $L^{\prime}$ have the signature, the discriminant, and the rank of $L$ and $L^{\prime}$ are respectively $(1,9)$, $\operatorname{discr} L=\operatorname{discr} L^{\prime}=-13$, and $\operatorname{rank} L=\operatorname{rank} L^{\prime}=10$, then, the lattices are primitive sublattices of the $K 3$ lattice and $U \oplus L^{\prime}$ is the orthogonal complement of $L$.

Proof Note that the discriminant groups $A_{L}, A_{L^{\prime}}$ of $L$ and $L^{\prime}$ are isomorphic to $\mathbb{Z} /$ $13 \mathbb{Z}$, and that the minimal number of the generators is $l\left(A_{L}\right)=l\left(A_{L^{\prime}}\right)=1$. Since the signature of $L$ and $L^{\prime}$ is $\left(t_{+}, t_{-}\right)=(1,9)$ and the $\operatorname{rank}$ is $\operatorname{rank} L=\operatorname{rank} L^{\prime}=10$, we have

$$
\begin{array}{r}
19-t_{-}=10 \geq 0, \quad 3-t_{+}=2 \geq 0, \quad \text { and } \\
22-\operatorname{rank} L=22-\operatorname{rank} L^{\prime}=12>1=l\left(A_{L}\right)=l\left(A_{L^{\prime}}\right),
\end{array}
$$

by Corollary 2.2, the first statement is shown. Since the discriminant of $U$ is -1 , we have $\operatorname{discr}\left(U \oplus L^{\prime}\right)=-\operatorname{discr} L^{\prime}=13=-\operatorname{discr} L$, and thus by Corollary 2.1, the last assertion is proved. 
Case 1 Set one-simplices of $\Sigma$ as follows:

$$
\begin{array}{lll}
v_{1}=(-1,-1,1), & v_{2}=(-1,-1,-1), & v_{3}=(2,-1,-1), \\
v_{4}=(2,0,-1), & v_{5}=(-1,2,-1), & v_{6}=(-1,1,0), \\
v_{7}=(0,-1,1), & v_{8}=(-1,-1,0), & v_{9}=(0,-1,-1), \\
v_{10}=(1,-1,-1), & v_{11}=(-1,0,-1), & v_{12}=(-1,1,-1), \\
v_{13}=(1,-1,0), &
\end{array}
$$

and let $\tilde{D}_{i}$ be the toric divisor determined by the lattice point $v_{i}$ for $i=1, \ldots, 13$, and $D_{i}:=\left.\tilde{D}_{i}\right|_{-K_{X}}$ with $X:=\widetilde{\mathbb{P}_{\Sigma}}$. It can be easily seen by formulas (3) and (2) that

$$
\begin{aligned}
& \rho_{\Delta}=13-3=10, D_{1}^{2}=D_{2}^{2}=D_{3}^{2}=-2, D_{4}^{2}=0, D_{5}^{2}=D_{6}^{2}=-2 \text {, } \\
& D_{7}^{2}=0, D_{8}^{2}=\cdots=D_{13}^{2}=-2 \text {. }
\end{aligned}
$$

Let $L$ be a lattice generated by divisors $\left\{D_{i}\right\}_{i=1}^{13}$. By solving the Eq. (4), one sees that $\left\{D_{7}, D_{4}, D_{3}, D_{13}, D_{10}, D_{9}, D_{2}, D_{8}, D_{11}, D_{12}\right\}$ form a basis for $L$. By taking a new basis

$$
\left\{\begin{array}{l}
D_{7}, D_{7}+D_{13}, D_{3}-D_{7}, D_{3}-D_{4}+3 D_{7}+D_{9}+D_{10}+2 D_{13}, \\
D_{10}, D_{2}+D_{8}+D_{9}, D_{2}, D_{8}, D_{11}, D_{12}
\end{array}\right\},
$$

one sees that the lattice $L$ is isometric to $U \oplus \tilde{L}$ with some lattice $\tilde{L}$. By a direct computation, one sees that $\operatorname{sgn} L=(1,9)$, discr $L=-13$, and $\operatorname{rank} L=10$, thus, $\operatorname{discr} \tilde{L}=13$ and rank $\tilde{L}=8$ hold. In particular, the discriminant group $A_{L}$ of $L$ is isomorphic to $\mathbb{Z} / 13 \mathbb{Z}$, and $l\left(A_{L}\right)=1$.

Set one-simplices of $\Sigma^{\prime}$ as follows:

$$
\begin{array}{lll}
m_{1}=(-1,-1,1), & m_{2}=(-1,-1,-1), & m_{3}=(2,-1,-1), \\
m_{4}=(2,0,-1), & m_{5}=(-1,1,0), & m_{6}=(0,-1,1), \\
m_{7}=(-1,0,-1), & m_{8}=(-1,-1,0), & m_{9}=(0,-1,-1), \\
m_{10}=(1,-1,-1), & m_{11}=(1,0,-1), & m_{12}=(0,0,-1), \\
m_{13}=(1,-1,0), &
\end{array}
$$

and let $\tilde{D}_{i}^{\prime}$ be the toric divisor determined by the lattice point $m_{i}$ for $i=1, \ldots, 13$, and $D_{i}^{\prime}:=\left.\tilde{D}_{i}^{\prime}\right|_{-K_{X}}$ with $X:=\widetilde{\mathbb{P}_{\Sigma^{\prime}}}$. It can be easily seen by formulas (3) and (2) that

$$
\begin{aligned}
\rho_{\Delta^{\prime}}=13-3=10, & D_{1}^{\prime 2}=D_{2}^{\prime 2}=D_{3}^{\prime 2}=-2, D_{4}^{\prime 2}=0, D_{5}^{\prime 2}=4, D_{6}^{\prime 2}=0, \\
& D_{7}^{\prime 2}=\cdots=D_{13}^{\prime 2}=-2 .
\end{aligned}
$$

Let $L^{\prime}$ be a lattice generated by divisors $\left\{D_{i}^{\prime}\right\}_{i=1}^{13}$. By solving the Eq. (4), one sees that $\left\{D_{4}^{\prime}, D_{6}^{\prime}, D_{13}^{\prime}, D_{3}^{\prime}, D_{10}^{\prime}, D_{9}^{\prime}, D_{2}^{\prime}, D_{8}^{\prime}, D_{7}^{\prime}, D_{12}^{\prime}\right\}$ form a basis for $L^{\prime}$. By taking a new basis

$$
\left\{\begin{array}{l}
D_{4}^{\prime}, D_{3}^{\prime}+D_{4}^{\prime}, D_{4}^{\prime}-D_{13}^{\prime}, 2 D_{3}^{\prime}+2 D_{4}^{\prime}-D_{6}^{\prime}+D_{10}^{\prime}+D_{13}^{\prime}, \\
-D_{4}^{\prime}+D_{10}^{\prime}, D_{9}^{\prime}, D_{2}^{\prime}, D_{8}^{\prime}, D_{7}^{\prime}, D_{12}^{\prime}
\end{array}\right\}
$$


one sees that the lattice $L^{\prime}$ is isometric to $U \oplus \tilde{L^{\prime}}$ with some lattice $\tilde{L}^{\prime}$. By a direct computation, one sees that $\operatorname{sgn} L^{\prime}=(1,9)$, discr $L^{\prime}=-13$, and rank $L^{\prime}=10$, thus, $\operatorname{discr} \tilde{L}^{\prime}=13$ and rank $\tilde{L}^{\prime}=8$ hold. In particular, the discriminant group $A_{L^{\prime}}$ of $L^{\prime}$ is isomorphic to $\mathbb{Z} / 13 \mathbb{Z}$, and $l\left(A_{L^{\prime}}\right)=1$.

Case 2 Set one-simplices of $\Sigma$ as follows:

$$
\begin{array}{lll}
v_{1}=(-1,-1,0), & v_{2}=(-1,-1,-1), & v_{3}=(3,-1,-1), \\
v_{4}=(2,0,-1), & v_{5}=(-1,2,-1), & v_{6}=(-1,1,0), \\
v_{7}=(0,-1,1), & v_{8}=(-1,0,0), & v_{9}=(0,-1,-1), \\
v_{10}=(1,-1,-1), & v_{11}=(2,-1,-1), & v_{12}=(-1,0,-1), \\
v_{13}=(-1,1,-1), &
\end{array}
$$

and let $\tilde{D}_{i}$ be the toric divisor determined by the lattice point $v_{i}$ for $i=1, \ldots, 13$, and $D_{i}:=\left.\tilde{D}_{i}\right|_{-K_{X}}$ with $X:=\widetilde{\mathbb{P}_{\Sigma}}$. It can be easily seen by formulas (3) and (2) that

$$
\rho_{\Delta}=13-3=10, \quad D_{1}^{2}=\cdots=D_{6}^{2}=-2, D_{7}^{2}=2, D_{8}^{2}=\cdots=D_{13}^{2}=-2 .
$$

Let $L$ be a lattice generated by divisors $\left\{D_{i}\right\}_{i=1}^{13}$. By solving the Eq. (4), one sees that $\left\{D_{7}, D_{1}, D_{8}, D_{2}, D_{12}, D_{13}, D_{9}, D_{10}, D_{11}, D_{3}\right\}$ form a basis for $L$. By taking a new basis

$$
\left\{\begin{array}{l}
D_{7}-D_{8}, D_{3}+D_{7}-D_{8}, D_{1}, D_{2}, D_{12}, D_{13}, D_{9} \\
D_{10},-D_{7}+D_{8}+D_{11},-2 D_{3}-4 D_{7}+5 D_{8}
\end{array}\right\}
$$

one sees that the lattice $L$ is isometric to $U \oplus \tilde{L}$ with some lattice $\tilde{L}$. By a direct computation, one sees that $\operatorname{sgn} L=(1,9)$, discr $L=-13$, and rank $L=10$, thus, $\operatorname{discr} \tilde{L}=13$ and rank $\tilde{L}=8$ hold. In particular, the discriminant group $A_{L}$ of $L$ is isomorphic to $\mathbb{Z} / 13 \mathbb{Z}$, and $l\left(A_{L}\right)=1$.

Set one-simplices of $\Sigma^{\prime}$ as follows:

$$
\begin{array}{lll}
m_{1}=(-1,-1,0), & m_{2}=(-1,-1,-1), & m_{3}=(3,-1,-1), \\
m_{4}=(2,0,-1), & m_{5}=(-1,1,0), & m_{6}=(0,-1,1), \\
m_{7}=(-1,0,-1), & m_{8}=(-1,0,0), & m_{9}=(0,-1,-1), \\
m_{10}=(1,-1,-1), & m_{11}=(2,-1,-1), & m_{12}=(0,0,-1), \\
m_{13}=(1,0,-1), &
\end{array}
$$

and let $\tilde{D}_{i}^{\prime}$ be the toric divisor determined by the lattice point $m_{i}$ for $i=1, \ldots, 13$, and $D_{i}^{\prime}:=\tilde{D}_{i}^{\prime} \mid-K_{X}$ with $X:=\widetilde{\mathbb{P}_{\Sigma^{\prime}}}$. It can be easily seen by formulas (3) and (2) that

$$
\begin{aligned}
& \rho_{\Delta^{\prime}}=13-3=10, D_{1}^{\prime 2}=\cdots=D_{4}^{\prime 2}=-2, D_{5}^{\prime 2}=4, D_{6}^{\prime 2}=2 \text {, } \\
& D_{7}^{\prime 2}=\cdots=D_{13}^{\prime 2}=-2 \text {. }
\end{aligned}
$$

Let $L^{\prime}$ be a lattice generated by divisors $\left\{D_{i}^{\prime}\right\}_{i=1}^{13}$. By solving the Eq. (4), one sees that $\left\{D_{6}^{\prime}, D_{1}^{\prime}, D_{8}^{\prime}, D_{4}^{\prime}, D_{3}^{\prime}, D_{11}^{\prime}, D_{10}^{\prime}, D_{9}^{\prime}, D_{13}^{\prime}, D_{12}^{\prime}\right\}$ form a basis for $L^{\prime}$. By taking a new 
basis

$$
\left\{\begin{array}{l}
D_{3}^{\prime}+D_{4}^{\prime}, D_{3}^{\prime}+D_{4}^{\prime}+D_{11}^{\prime},-4 D_{3}^{\prime}-4 D_{4}^{\prime}+D_{6}^{\prime}-2 D_{11}^{\prime}, \\
-D_{4}^{\prime}, D_{1}^{\prime}, D_{8}^{\prime},-D_{3}^{\prime}-D_{4}^{\prime}+D_{10}^{\prime}, D_{9}^{\prime}, \\
-2 D_{3}^{\prime}-2 D_{4}^{\prime}-D_{11}^{\prime}+D_{13}^{\prime}, D_{12}^{\prime}
\end{array}\right\}
$$

one sees that the lattice $L^{\prime}$ is isometric to $U \oplus \tilde{L^{\prime}}$ with some lattice $\tilde{L}^{\prime}$. By a direct computation, one sees that $\operatorname{sgn} L^{\prime}=(1,9)$, discr $L^{\prime}=-13$, and rank $L^{\prime}=10$, thus, $\operatorname{discr} \tilde{L}^{\prime}=13$ and rank $\tilde{L}^{\prime}=8$ hold. In particular, the discriminant group $A_{L^{\prime}}$ of $L^{\prime}$ is isomorphic to $\mathbb{Z} / 13 \mathbb{Z}$, and $l\left(A_{L^{\prime}}\right)=1$.

$\underline{\text { Case } 3}$ Set one-simplices of $\Sigma$ as follows:

$$
\begin{array}{lll}
v_{1}=(-1,-1,1), & v_{2}=(-1,-1,-1), & v_{3}=(3,-1,-1) \\
v_{4}=(2,0,-1), & v_{5}=(-1,1,0), & v_{6}=(0,-1,1), \\
v_{7}=(-1,0,-1), & v_{8}=(-1,-1,0), & v_{9}=(0,-1,-1), \\
v_{10}=(1,-1,-1), & v_{11}=(2,-1,-1), & v_{12}=(0,0,-1), \\
v_{13}=(1,0,-1), &
\end{array}
$$

and let $\tilde{D}_{i}$ be the toric divisor determined by the lattice point $v_{i}$ for $i=1, \ldots, 13$, and $D_{i}:=\left.\tilde{D}_{i}\right|_{-K_{X}}$ with $X:=\widetilde{\mathbb{P}_{\Sigma}}$. It can be easily seen by formulas (3) and (2) that

$$
\begin{gathered}
\rho_{\Delta}=13-3=10, \\
D_{1}^{2}=\cdots=D_{4}^{2}=-2, D_{5}^{2}=4, D_{6}^{2}=0, \\
D_{7}^{2}=\cdots=D_{13}^{2}=-2 .
\end{gathered}
$$

Let $L$ be a lattice generated by divisors $\left\{D_{i}\right\}_{i=1}^{13}$. By solving the Eq. (4), one sees that $\left\{D_{8}, D_{1}, D_{6}, D_{4}, D_{13}, D_{12}, D_{3}, D_{11}, D_{10}, D_{9}\right\}$ form a basis for $L$. By taking a new basis

$$
\left\{\begin{array}{l}
D_{6}, D_{4}+D_{6}, D_{1}-D_{3}-D_{4}, D_{8},-D_{6}+D_{13} \\
D_{12}, D_{3}-D_{4}-4 D_{6}, D_{11}, D_{10}, D_{9}
\end{array}\right\}
$$

one sees that the lattice $L$ is isometric to $U \oplus \tilde{L}$ with some lattice $\tilde{L}$. By a direct computation, one sees that $\operatorname{sgn} L=(1,9)$, discr $L=-13$, and $\operatorname{rank} L=10$, thus, $\operatorname{discr} \tilde{L}=13$ and $\operatorname{rank} \tilde{L}=8$ hold. In particular, the discriminant group $A_{L}$ of $L$ is isomorphic to $\mathbb{Z} / 13 \mathbb{Z}$, and $l\left(A_{L}\right)=1$.

Set one-simplices of $\Sigma^{\prime}$ as follows:

$$
\begin{array}{lll}
m_{1}=(-1,-1,0), & m_{2}=(-1,-1,-1), & m_{3}=(2,-1,-1), \\
m_{4}=(2,0,-1), & m_{5}=(-1,2,-1), & m_{6}=(-1,1,0), \\
m_{7}=(0,-1,1), & m_{8}=(-1,0,0), & m_{9}=(0,-1,-1), \\
m_{10}=(1,-1,-1), & m_{11}=(1,-1,0), & m_{12}=(-1,0,-1), \\
m_{13}=(-1,1,-1), &
\end{array}
$$


and let $\tilde{D}_{i}^{\prime}$ be the toric divisor determined by the lattice point $m_{i}$ for $i=1, \ldots, 13$, and $D_{i}^{\prime}:=\tilde{D}_{i}^{\prime} \mid-K_{X}$ with $X:=\widetilde{\mathbb{P}_{\Sigma^{\prime}}}$. It can be easily seen by formulas (3) and (2) that

$$
\begin{gathered}
\rho_{\Delta^{\prime}}=13-3=10, D_{1}^{\prime 2}=D_{2}^{\prime 2}=D_{3}^{\prime 2}=-2, D_{4}^{\prime 2}=0, D_{5}^{\prime 2}=D_{6}^{\prime 2}=-2, \\
D_{7}^{\prime 2}=2, D_{8}^{\prime 2}=\cdots=D_{13}^{\prime 2}=-2 .
\end{gathered}
$$

Let $L^{\prime}$ be a lattice generated by divisors $\left\{D_{i}^{\prime}\right\}_{i=1}^{13}$. By solving the Eq. (4), one sees that $\left\{D_{7}^{\prime}, D_{4}^{\prime}, D_{3}^{\prime}, D_{11}^{\prime}, D_{10}^{\prime}, D_{9}^{\prime}, D_{2}^{\prime}, D_{1}^{\prime}, D_{12}^{\prime}, D_{13}^{\prime}\right\}$ form a basis for $L^{\prime}$. By taking a new basis

$$
\left\{\begin{array}{l}
D_{3}^{\prime}+D_{4}^{\prime}, D_{4}^{\prime},-2 D_{3}^{\prime}-4 D_{4}^{\prime}+D_{7}^{\prime},-D_{4}^{\prime}+D_{11}^{\prime}, \\
-D_{4}^{\prime}+D_{10}^{\prime}, D_{9}^{\prime}, D_{2}^{\prime}, D_{1}^{\prime}, D_{12}^{\prime}, D_{13}^{\prime}
\end{array}\right\}
$$

one sees that the lattice $L^{\prime}$ is isometric to $U \oplus \tilde{L^{\prime}}$ with some lattice $\tilde{L}^{\prime}$. By a direct computation, one sees that $\operatorname{sgn} L^{\prime}=(1,9)$, discr $L^{\prime}=-13$, and rank $L^{\prime}=10$, thus, $\operatorname{discr} \tilde{L}^{\prime}=13$ and rank $\tilde{L}^{\prime}=8$ hold. In particular, the discriminant group $A_{L^{\prime}}$ of $L^{\prime}$ is isomorphic to $\mathbb{Z} / 13 \mathbb{Z}$, and $l\left(A_{L^{\prime}}\right)=1$.

Case 4 Set one-simplices of $\Sigma$ as follows:

$$
\begin{aligned}
& v_{1}=(-1,-1,1), \quad v_{2}=(-1,-1,-1), v_{3}=(3,-1,-1) \text {, } \\
& v_{4}=(2,0,-1), \quad v_{5}=(-1,2,-1), \quad v_{6}=(-1,1,0) \text {, } \\
& v_{7}=(0,-1,1), \quad v_{8}=(-1,-1,0), \quad v_{9}=(0,-1,-1) \text {, } \\
& v_{10}=(1,-1,-1), v_{11}=(2,-1,-1), \quad v_{12}=(-1,0,-1) \text {, } \\
& v_{13}=(-1,1,-1) \text {, }
\end{aligned}
$$

and let $\tilde{D}_{i}$ be the toric divisor determined by the lattice point $v_{i}$ for $i=1, \ldots, 13$, and $D_{i}:=\left.\tilde{D}_{i}\right|_{-K_{X}}$ with $X:=\widetilde{\mathbb{P}_{\Sigma}}$. One can easily seen by formulas (3) and (2) that

$$
\begin{gathered}
\rho_{\Delta}=13-3=10, D_{1}=\cdots=D_{6}=-2, D_{7}=0, \\
D_{8}=\cdots=D_{13}=-2 .
\end{gathered}
$$

Let $L$ be a lattice generated by divisors $\left\{D_{i}\right\}_{i=1}^{13}$. By solving the Eq. (4), one sees that $\left\{D_{7}, D_{4}, D_{3}, D_{11}, D_{10}, D_{9}, D_{2}, D_{12}, D_{13}, D_{8}\right\}$ form a basis for $L$. By taking a new basis

$$
\left\{D_{7}, D_{4}+D_{7}, D_{3}-D_{4}-4 D_{7}, D_{11}, D_{10}, D_{9}, D_{2}, D_{12}, D_{13}, D_{8}\right\}
$$

one sees that the lattice $L$ is isometric to $U \oplus \tilde{L}$ with some lattice $\tilde{L}$. By a direct computation, one sees that $\operatorname{sgn}_{\tilde{L}} L=(1,9)$, $\operatorname{discr} L=-13$, and $\operatorname{rank} L=10$, and thus, discr $\tilde{L}=13$ and $\operatorname{rank} \tilde{L}=8$ hold. In particular, the discriminant group $A_{L}$ of $L$ is isomorphic to $\mathbb{Z} / 13 \mathbb{Z}$, and $l\left(A_{L}\right)=1$. 
Set one-simplices of $\Sigma^{\prime}$ as follows:

$$
\begin{array}{lll}
m_{1}=(-1,-1,0), & m_{2}=(-1,-1,-1), & m_{3}=(2,-1,-1), \\
m_{4}=(2,0,-1), & m_{5}=(-1,1,0), & m_{6}=(0,-1,1), \\
m_{7}=(-1,0,-1), & m_{8}=(0,-1,-1), & m_{9}=(1,-1,-1), \\
m_{10}=(-1,0,0), & m_{11}=(1,-1,0), & m_{12}=(0,0,-1), \\
m_{13}=(1,0,-1), &
\end{array}
$$

and let $\tilde{D}_{i}^{\prime}$ be the toric divisor determined by the lattice point $m_{i}$ for $i=1, \ldots, 13$, and $D_{i}^{\prime}:=\left.\tilde{D}_{i}^{\prime}\right|_{-K_{X}}$ with $X:=\widetilde{\mathbb{P}_{\Sigma^{\prime}}}$. It can be easily seen by formulas (3) and (2) that

$$
\begin{aligned}
& \rho_{\Delta^{\prime}}=13-3=10, D_{1}^{\prime 2}=D_{2}^{\prime 2}=D_{3}^{\prime 2}=-2, D_{4}^{\prime 2}=0, D_{5}^{\prime 2}=4 \text {, } \\
& D_{6}^{\prime 2}=2, D_{7}^{\prime 2}=\cdots=D_{13}^{\prime 2}=-2 \text {. }
\end{aligned}
$$

Let $L^{\prime}$ be a lattice generated by divisors $\left\{D_{i}^{\prime}\right\}_{i=1}^{13}$. By solving the Eq. (4), one sees that $\left\{D_{11}^{\prime}, D_{3}^{\prime}, D_{4}^{\prime}, D_{13}^{\prime}, D_{12}^{\prime}, D_{7}^{\prime}, D_{2}^{\prime}, D_{8}^{\prime}, D_{1}^{\prime}, D_{10}^{\prime}\right\}$ form a basis for $L^{\prime}$. By taking a new basis

$$
\left\{\begin{array}{l}
D_{4}^{\prime}, D_{4}^{\prime}+D_{13}^{\prime}, D_{11}^{\prime}, D_{3}^{\prime}-2 D_{4}^{\prime}-D_{13}^{\prime}, \\
-D_{4}^{\prime}+D_{12}^{\prime}, D_{7}^{\prime}, D_{2}^{\prime}, D_{8}^{\prime}, D_{1}^{\prime}, D_{10}^{\prime}
\end{array}\right\},
$$

one sees that the lattice $L^{\prime}$ is isometric to $U \oplus \tilde{L^{\prime}}$ with some lattice $\tilde{L}^{\prime}$. By a direct computation, one sees that $\operatorname{sgn} L^{\prime}=(1,9)$, discr $L^{\prime}=-13$, and $\operatorname{rank} L^{\prime}=10$, thus, $\operatorname{discr} \tilde{L}^{\prime}=13$ and rank $\tilde{L}^{\prime}=8$ hold. In particular, the discriminant group $A_{L^{\prime}}$ of $L^{\prime}$ is isomorphic to $\mathbb{Z} / 13 \mathbb{Z}$, and $l\left(A_{L^{\prime}}\right)=1$.

In all cases 1 to 4 , we obtain lattices $L$ and $L^{\prime}$ satisfying assumptions in Lemma 3.3. Therefore, we can conclude that $\operatorname{Pic}_{\Delta}=U \oplus \tilde{L}$, $\operatorname{Pic}_{\Delta^{\prime}}=U \oplus \tilde{L^{\prime}}$, with discr $\tilde{L}=$ $\operatorname{discr} \tilde{L}^{\prime}=13$ and $\operatorname{rank} \tilde{L}=\operatorname{rank} \tilde{L}^{\prime}=8$, and that the relation $\left(\operatorname{Pic}_{\Delta}\right){ }_{\Lambda_{K 3}}^{\perp} \simeq U \oplus \operatorname{Pic}_{\Delta^{\prime}}$ holds.

\subsection{Nos. 35, 36, and 37}

Set one-simplices of $\Sigma$ in terms of a basis of $M_{(1,1,4,6)} \otimes \mathbb{R}$

$$
\begin{aligned}
& (-1,1,0,0),(-4,0,1,0),(-6,0,0,1): \\
& v_{1}=(-1,-1,1), \quad v_{2}=(-1,-1,-1), v_{3}=(11,-1,-1) \text {, } \\
& v_{4}=(-1,2,-1), \quad v_{5}=(-1,-1,0), \quad v_{6}=(5,-1,0) \text {, } \\
& v_{7}=(0,-1,-1), \quad v_{8}=(1,-1,-1), \quad v_{9}=(2,-1,-1) \text {, } \\
& v_{10}=(3,-1,-1), v_{11}=(4,-1,-1), \quad v_{12}=(5,-1,-1) \text {, } \\
& v_{13}=(6,-1,-1), v_{14}=(7,-1,-1), \quad v_{15}=(8,-1,-1) \text {, } \\
& v_{16}=(9,-1,-1), v_{17}=(10,-1,-1), v_{18}=(7,0,-1) \text {, } \\
& v_{19}=(3,1,-1), \quad v_{20}=(-1,0,-1), \quad v_{21}=(-1,1,-1) \text {, }
\end{aligned}
$$


and let $\tilde{D}_{i}$ be the toric divisor determined by the lattice point $v_{i}$ for $i=1, \ldots, 21$, and $D_{i}:=\left.\tilde{D}_{i}\right|_{-K_{X}}$ with $X:=\widetilde{\mathbb{P}_{\Sigma}}$. It can be easily seen by formulas (3) and (2) that

$$
\begin{gathered}
\rho_{\Delta}=21-3=18, D_{1}^{2}=2, D_{2}^{2}=D_{3}^{2}=-2, D_{4}^{2}=0 \\
D_{5}^{2}=\cdots=D_{21}^{2}=-2 .
\end{gathered}
$$

Let $L$ be a lattice generated by divisors $\left\{D_{i}\right\}_{i=1}^{21}$. By solving the Eq. (4), one sees that $\left\{D_{4}, D_{1}, D_{5}, D_{21}, D_{20}, D_{19}, D_{18}, D_{3}, D_{17}, D_{16}, D_{15}, D_{14}, D_{13}\right.$, $\left.D_{12}, D_{11}, D_{10}, D_{9}, D_{8}\right\}$ form a basis for $L$. By taking a new basis

$$
\left\{\begin{array}{l}
D_{4}, D_{4}+D_{21},-D_{5},-D_{1}+2 D_{4}+D_{19}+D_{21},-D_{4}+D_{20} \\
D_{3}-D_{4}+D_{16}+D_{17}+D_{18}+D_{19}-D_{20}-D_{21}, D_{18}, D_{3} \\
D_{17}, D_{14}+D_{15}+D_{16}, D_{15}, D_{14}, D_{13}+D_{14}+D_{15} \\
D_{12}, D_{11}, D_{10}, D_{9}, D_{8}
\end{array}\right\}
$$

one sees that the lattice $L$ is isometric to $U \oplus \tilde{L}$, where $\tilde{L}$ is a negative-definite of rank 16 and discriminant 1 . By the classification of unimodular lattices, we have $\tilde{L} \simeq E_{8}^{\oplus 2}$. Therefore, $\mathrm{Pic}_{\Delta} \simeq U \oplus E_{8}^{\oplus 2}$.

Set one-simplices of $\Sigma^{\prime}$ in terms of a basis of $M_{(3,5,11,14)} \otimes \mathbb{R}$

$$
\begin{aligned}
& (1,0,1,-1),(2,1,-1,0),(10,-1,-1,-1): \\
& m_{1}=(-1,0,0), m_{2}=(0,0,1), m_{3}=(2,4,-1), \\
& m_{4}=(1,-1,0), m_{5}=(1,2,0),
\end{aligned}
$$

and let $\tilde{D}_{i}^{\prime}$ be the toric divisor determined by the lattice point $m_{i}$ for $i=1, \ldots, 5$, and $D_{i}^{\prime}:=\left.\tilde{D}_{i}^{\prime}\right|_{-K_{X}}$ with $X:=\widetilde{\mathbb{P}_{\Sigma^{\prime}}}$. It can be easily seen by formulas (3) and (2) that

$$
\rho_{\Delta^{\prime}}=5-3=2, \quad D_{1}^{\prime 2}=18, D_{2}^{\prime 2}=D_{3}^{\prime 2}=0, D_{4}^{\prime 2}=8, D_{5}^{\prime 2}=-2 .
$$

Let $L^{\prime}$ be a lattice generated by divisors $\left\{D_{i}^{\prime}\right\}_{i=1}^{5}$. By solving the Eq. (4), one sees that $\left\{D_{3}^{\prime}, D_{5}^{\prime}\right\}$ form a basis for $L^{\prime}$. By taking a new basis $\left\{D_{3}^{\prime}, D_{3}^{\prime}+D_{5}^{\prime}\right\}$, one sees that the lattice $L^{\prime}$ is isometric to $U$, which is a hyperbolic primitive sublattice of the $K 3$ lattice. Thus, $\mathrm{Pic}_{\Delta^{\prime}} \simeq U$.

It is well-known that lattices $U$ and $U \oplus E_{8}^{\oplus 2}$ are primitive sublattices of the $K 3$ lattice $\Lambda_{K 3}$ and it is clear that the relation $\left(\mathrm{Pic}_{\Delta}\right){ }_{\Lambda_{K 3}}^{\perp} \simeq U_{U^{\oplus 3}}^{\perp} \oplus\left(E_{8}^{\oplus 2}\right)_{E_{8}^{\oplus 3}}^{\perp}=U^{\oplus 2} \simeq$ $U \oplus$ Pic $_{\Delta^{\prime}}$ holds.

\subsection{Nos. 38 and 40}

Take bases of $M_{(1,1,3,5)} \otimes \mathbb{R}$, and of $M_{(3,4,10,13)} \otimes \mathbb{R}$, respectively:

$$
\begin{array}{r}
\{(-1,1,0,0),(-3,0,1,0),(-5,0,0,1)\}, \\
\{(1,0,1,-1),(3,1,0,-1),(9,-1,-1,-1)\} .
\end{array}
$$


Lemma 3.4 If $L$ is a negative-definite lattice of rank 15 of discriminant -2 , then, it is a primitive sublattice of the $K 3$ lattice.

Proof Note that the discriminant group of $L$ is isomorphic to $\mathbb{Z} / 2 \mathbb{Z}$ of number of generator $l\left(A_{L}\right)=1$. Since the signature of $L$ and $L^{\prime}$ is $\left(t_{+}, t_{-}\right)=(0,15)$ and the rank is $\operatorname{rank} L=15$, we have

$$
19-t_{-}=4 \geq 0, \quad 3-t_{+}=3 \geq 0, \quad \text { and } 22-\operatorname{rank} L=7>1=l\left(A_{L}\right),
$$

by Corollary 2.2, the assertion is proved.

Case 1 Set one-simplices of $\Sigma$ as follows:

$$
\begin{array}{lll}
v_{1}=(-1,-1,1), & v_{2}=(-1,-1,-1), & v_{3}=(9,-1,-1), \\
v_{4}=(0,2,-1), & v_{5}=(-1,2,-1), & v_{6}=(-1,-1,0), \\
v_{7}=(0,-1,-1), & v_{8}=(1,-1,-1), & v_{9}=(2,-1,-1), \\
v_{10}=(3,-1,-1), & v_{11}=(4,-1,-1), & v_{12}=(5,-1,-1), \\
v_{13}=(6,-1,-1), & v_{14}=(7,-1,-1), & v_{15}=(8,-1,-1), \\
v_{16}=(6,0,-1), & v_{17}=(3,1,-1), & v_{18}=(-1,0,-1), \\
v_{19}=(-1,1,-1), & v_{20}=(4,-1,0),
\end{array}
$$

and let $\tilde{D}_{i}$ be the toric divisor determined by the lattice point $v_{i}$ for $i=1, \ldots, 20$, and $D_{i}:=\left.\tilde{D}_{i}\right|_{-K_{X}}$ with $X:=\widetilde{\mathbb{P}_{\Sigma}}$. It can be easily seen by formulas (3) and (2) that

$$
\rho_{\Delta}=20-3=17, \quad D_{1}^{2}=2, \quad D_{2}^{2}=\cdots=D_{20}^{2}=-2 .
$$

Let $L$ be a lattice generated by divisors $\left\{D_{i}\right\}_{i=1}^{20}$. By solving the Eq. (4), one sees that $\left\{D_{4}, D_{5}, D_{19}, D_{18}, D_{2}, D_{1}, D_{20}, D_{3}, D_{16}, D_{15}, D_{14}, D_{13}, D_{12}, D_{11}, D_{10}, D_{9}, D_{8}\right\}$ form a basis for $L$. By taking a new basis

$$
\left\{\begin{array}{l}
D_{4}+D_{5}+D_{19}, D_{4}+D_{5}, D_{4}, D_{1}-4 D_{4}-4 D_{5}-2 D_{19}, \\
D_{2},-D_{4}-D_{5}+D_{18}, D_{20}, D_{3}, D_{16}, D_{15}, D_{14}, D_{13}, \\
D_{12}, D_{11}, D_{10}, D_{9}, D_{8}
\end{array}\right\}
$$

one sees that the lattice $L$ is isometric to $U \oplus \tilde{L}$, where discr $\tilde{L}=-2$ and $\operatorname{rank} \tilde{L}=15$.

Set one-simplices of $\Sigma^{\prime}$ as follows:

$$
\begin{aligned}
& m_{1}=(1,0,0), \quad m_{2}=(0,1,0), \quad m_{3}=(0,0,1), \\
& m_{4}=(0,-2,-3), m_{5}=(-1,-3,-5), m_{6}=(0,-1,-1),
\end{aligned}
$$

and let $\tilde{D}_{i}^{\prime}$ be the toric divisor determined by the lattice point $m_{i}$ for $i=1, \ldots, 6$, and $D_{i}^{\prime}:=\left.\tilde{D}_{i}^{\prime}\right|_{-K_{X}}$ with $X:=\widetilde{\mathbb{P}_{\Sigma^{\prime}}}$. It can be easily seen by formulas (3) and (2) that

$$
\begin{aligned}
\rho_{\Delta}=6-3=3, D_{1}^{\prime 2} & =0, D_{2}^{\prime 2}=6, D_{3}^{\prime 2}=16, \\
D_{4}^{\prime 2} & =-2, D_{5}^{\prime 2}=0, D_{6}^{\prime 2}=-2 .
\end{aligned}
$$


Let $L^{\prime}$ be a lattice generated by divisors $\left\{D_{i}^{\prime}\right\}_{i=1}^{6}$. By solving the Eq. (4), one sees that $\left\{D_{1}^{\prime}, D_{4}^{\prime}, D_{6}^{\prime}\right\}$ form a basis for $L^{\prime}$. By taking a new basis $\left\{D_{1}^{\prime}, D_{1}^{\prime}+D_{4}^{\prime}, D_{6}^{\prime}-D_{1}^{\prime}\right\}$, one sees that the lattice $L^{\prime}$ is isometric to $U \oplus A_{1}$, which is hyperbolic and a primitive sublattice of the $K 3$ lattice. Therefore, $\operatorname{Pic}_{\Delta^{\prime}} \simeq U \oplus A_{1}$. Note that the discriminant group of $\mathrm{Pic}_{\Delta^{\prime}}$ is isomorphic to $\mathbb{Z} / 2 \mathbb{Z}$ since 2 is a prime number.

Case 2 Set one-simplices of $\Sigma$ as follows:

$$
\begin{array}{lll}
v_{1}=(-1,-1,-1), & v_{2}=(1,1,3), & v_{3}=(1,3,9), \\
v_{4}=(1,3,-1), & v_{5}=(1,0,-1), & v_{6}=(0,0,1), \\
v_{7}=(0,1,4), & v_{8}=(0,1,-1), & v_{9}=(1,2,6), \\
v_{10}=(1,2,-1), & v_{11}=(1,1,-1), & v_{12}=(1,3,8), \\
v_{13}=(1,3,7), & v_{14}=(1,3,6), & v_{15}=(1,3,5), \\
v_{16}=(1,3,4), & v_{17}=(1,3,3), & v_{18}=(1,3,2), \\
v_{19}=(1,3,1), & v_{20}=(1,3,0), &
\end{array}
$$

and let $\tilde{D}_{i}$ be the toric divisor determined by the lattice point $v_{i}$ for $i=1, \ldots, 20$, and $D_{i}:=\tilde{D}_{i} \mid-K_{X}$ with $X:=\widetilde{\mathbb{P}_{\Sigma}}$. It can be easily seen by formulas (3) and (2) that

$$
\begin{aligned}
& \rho_{\Delta}=20-3=17, D_{1}^{2}=2, D_{2}^{2}=\cdots=D_{4}^{2}=-2 \\
& D_{5}^{2}=0, D_{6}^{2}=\cdots=D_{20}^{2}=-2 .
\end{aligned}
$$

Let $L$ be a lattice generated by divisors $\left\{D_{i}\right\}_{i=1}^{20}$. By solving the Eq. (4), one sees that $\left\{D_{5}, D_{1}, D_{11}, D_{2}, D_{9}, D_{3}, D_{12}, D_{13}, D_{14}, D_{15}, D_{16}, D_{17}, D_{18}, D_{19}, D_{20}, D_{4}, D_{8}\right\}$ form a basis for $L$. By taking a new basis

$$
\left\{\begin{array}{l}
D_{5}, D_{5}+D_{11}, D_{2}-2 D_{5}-D_{11},-D_{1}+D_{2}+2 D_{5}+D_{11}, \\
D_{9}, D_{3}, D_{12}, D_{13}, D_{14}, D_{15}, D_{16}, D_{17}, D_{18}, D_{19}, D_{20}, D_{4}, D_{8}
\end{array}\right\},
$$

one sees that the lattice $L$ is isometric to $U \oplus \tilde{L}$, where $\tilde{L}$ is of rank 15 and of discriminant -2 .

Set one-simplices of $\Sigma^{\prime}$ as follows:

$$
\begin{aligned}
& m_{1}=(-1,0,0), \quad m_{2}=(2,-1,0), \quad m_{3}=(0,0,1), \\
& m_{4}=(-2,4,-1), m_{5}=(-1,3,-1), m_{6}=(-1,2,0),
\end{aligned}
$$

and let $\tilde{D}_{i}^{\prime}$ be the toric divisor determined by the lattice point $m_{i}$ for $i=1, \ldots, 6$, and $D_{i}^{\prime}:=\left.\tilde{D}_{i}^{\prime}\right|_{-K_{X}}$ with $X:=\widetilde{\mathbb{P}_{\Sigma^{\prime}}}$. It can be easily seen by formulas (3) and (2) that

$$
\begin{aligned}
& \rho_{\Delta^{\prime}}=6-3=3, D_{1}^{\prime 2}=16, D_{2}^{\prime 2}=6, D_{3}^{\prime 2}=0, \\
& D_{4}^{\prime 2}=D_{5}^{\prime 2}=D_{6}^{\prime 2}=-2 \text {. }
\end{aligned}
$$

Let $L^{\prime}$ be a lattice generated by divisors $\left\{D_{i}^{\prime}\right\}_{i=1}^{6}$. By solving the Eq. (4), one sees that $\left\{D_{3}^{\prime}, D_{6}^{\prime}, D_{5}^{\prime}\right\}$ form a basis for $L^{\prime}$, with respect to which the intersection matrix of $L^{\prime}$ is given by $\left(\begin{array}{ccc}0 & 1 & 0 \\ 1 & -2 & 0 \\ 0 & 0 & -2\end{array}\right)$. By taking a new basis $\left\{D_{3}^{\prime}, D_{3}^{\prime}+D_{6}^{\prime}, D_{5}^{\prime}\right\}$, one sees that 
the lattice $L^{\prime}$ is isometric to $U \oplus A_{1}$, which is a primitive sublattice of the $K 3$ lattice. Therefore, $\mathrm{Pic}_{\Delta^{\prime}} \simeq U \oplus A_{1}$.

In cases 1 and 2 , we obtain a lattice $L \simeq U \oplus \tilde{L}$, where $\tilde{L}$ is a lattice satisfying the assumption of Lemma 3.4. Therefore, $L$ is a primitive sublattice of the $K 3$ lattice, and that $\mathrm{Pic}_{\Delta}=L$ holds. Since discr Pic $\operatorname{Pi}_{\Delta}=\operatorname{discr}\left(U \oplus \operatorname{Pic}_{\Delta^{\prime}}\right)=2$, by Corollary 2.1, the relation $\left(\mathrm{Pic}_{\Delta}\right){ }_{\Lambda_{K 3}}^{\perp} \simeq U \oplus \mathrm{Pic}_{\Delta^{\prime}}$ holds. Moreover, by Lemma 2.1, we have $\mathrm{Pic}_{\Delta} \simeq$ $\left(U^{\oplus 2} \oplus A_{1}\right)_{\Lambda_{K 3}}^{\perp} \simeq U \oplus E_{7} \oplus E_{8}$.

\subsection{Nos. 41, 42, and 43}

Set one-simplices of $\Sigma$ in terms of a basis of $M_{(1,1,3,4)} \otimes \mathbb{R}$

$$
\begin{aligned}
& (-1,1,0,0),(-3,0,1,0),(-4,0,0,1): \\
& v_{1}=(-1,2,-1), \quad v_{2}=(-1,-1,-1), v_{3}=(8,-1,-1) \text {, } \\
& v_{4}=(0,-1,1), \quad v_{5}=(-1,-1,1), \quad v_{6}=(2,1,-1) \text {, } \\
& v_{7}=(5,0,-1), \quad v_{8}=(0,-1,-1), \quad v_{9}=(1,-1,-1) \text {, } \\
& v_{10}=(2,-1,-1), v_{11}=(3,-1,-1), \quad v_{12}=(4,-1,-1) \text {, } \\
& v_{13}=(5,-1,-1), v_{14}=(6,-1,-1), \quad v_{15}=(7,-1,-1) \text {, } \\
& v_{16}=(4,-1,0), \quad v_{17}=(-1,-1,0), \quad v_{18}=(-1,1,-1) \text {, } \\
& v_{19}=(-1,0,-1) \text {, }
\end{aligned}
$$

and let $\tilde{D}_{i}$ be the toric divisor determined by the lattice point $v_{i}$ for $i=1, \ldots, 19$, and $D_{i}:=\left.\tilde{D}_{i}\right|_{-K_{X}}$ with $X:=\widetilde{\mathbb{P}_{\Sigma}}$. It can be easily seen by formulas (3) and (2) that

$$
\rho_{\Delta}=19-3=16, \quad D_{1}^{2}=0, \quad D_{2}^{2}=\cdots=D_{19}=-2 .
$$

Let $L$ be a lattice generated by divisors $\left\{D_{i}\right\}_{i=1}^{19}$. By solving the Eq. (4), one sees that $\left\{D_{6}, D_{1}, D_{4}, D_{18}, D_{19}, D_{2}, D_{8}, D_{9}, D_{10}, D_{11}, D_{17}, D_{5}, D_{16}, D_{3}, D_{15}, D_{14}\right\}$ form a basis for $L$. By taking a new basis

$$
\left\{\begin{array}{l}
D_{1}+D_{4}, D_{1}, D_{1}-D_{4}-D_{5}+D_{6}-D_{17}+D_{18}, D_{17}, D_{19}, \\
D_{2}+D_{19}, D_{8}, D_{9}, D_{10}, D_{11}, \\
3 D_{1}+D_{2}-D_{5}+D_{8}+D_{9}+D_{10}+D_{11}+D_{18}+D_{19}, \\
-3 D_{1}+D_{5}-D_{6},-D_{1}+D_{16}, D_{3}, D_{15}, D_{14}
\end{array}\right\},
$$

one sees that the lattice $L$ is isometric to $U \oplus \tilde{L}$ with some lattice $\tilde{L}$. By a direct computation, one sees that $\operatorname{sgn} L=\left(t_{+}, t_{-}\right)=(1,15)$, discr $L=-3$, and $\operatorname{rank} L=$ 16 , and thus, discr $\tilde{L}=3$ and $\operatorname{rank} \tilde{L}=14$ hold. In particular, the discriminant group $A_{L}$ of $L$ is isomorphic to $\mathbb{Z} / 3 \mathbb{Z}$, and $l\left(A_{L}\right)=1$. Therefore, one observes that

$$
19-t_{-}=4 \geq 0, \quad 3-t_{+}=2 \geq 0, \quad 22-\operatorname{rank} L=6>1=l\left(A_{L}\right)
$$

and by Corollary 2.2, $L$ is a primitive sublattice of the $K 3$ lattice. Therefore, $\mathrm{Pic}_{\Delta} \simeq$ $U \oplus \tilde{L}$ with discr $\tilde{L}=3$ and $\operatorname{rank} \tilde{L}=14$. 
Set one-simplices of $\Sigma^{\prime}$ in terms of a basis of $M_{(3,4,11,18)} \otimes \mathbb{R}$

$$
\begin{aligned}
& (-1,8,-1,-1),(0,-1,2,-1),(-1,-1,-1,1): \\
& m_{1}=(1,0,0), \quad m_{2}=(0,1,0), \quad m_{3}=(0,0,1), \\
& m_{4}=(-1,-3,-4), m_{5}=(0,-2,-3), m_{6}=(0,0,-1), \\
& m_{7}=(0,-1,-2),
\end{aligned}
$$

and let $\tilde{D}_{i}^{\prime}$ be the toric divisor determined by the lattice point $m_{i}$ for $i=1, \ldots, 7$, and $D_{i}^{\prime}:=\left.\tilde{D}_{i}^{\prime}\right|_{-K_{X}}$ with $X:=\widetilde{\mathbb{P}_{\Sigma^{\prime}}}$. It can be easily seen by formulas (3) and (2) that

$$
\begin{aligned}
& \rho_{\Delta^{\prime}}=7-3=4, D_{1}^{\prime 2}=0, D_{2}^{\prime 2}=6, D_{3}^{\prime 2}=12 \\
& D_{4}^{\prime 2}=0, D_{5}^{\prime 2}=D_{6}^{\prime 2}=D_{7}^{\prime 2}=-2 .
\end{aligned}
$$

Let $L^{\prime}$ be a lattice generated by divisors $\left\{D_{i}^{\prime}\right\}_{i=1}^{7}$. By solving the Eq. (4), one sees that $\left\{D_{4}^{\prime}, D_{5}^{\prime}, D_{7}^{\prime}, D_{6}^{\prime}\right\}$ form a basis for $L^{\prime}$. By taking a new basis $\left\{D_{4}^{\prime}, D_{4}^{\prime}+D_{5}^{\prime},-D_{4}^{\prime}+D_{7}^{\prime}, D_{6}^{\prime}\right\}$, one sees that the lattice $L^{\prime}$ is isometric to $U \oplus A_{2}$, which is a primitive sublattice of the $K 3$ lattice. Therefore, $\operatorname{Pic}_{\Delta^{\prime}} \simeq U \oplus A_{2}$.

Since discr Pic $\operatorname{Pi}_{\Delta}=\operatorname{discr} U \oplus \tilde{L}=-\operatorname{discr} U^{\oplus 2} \oplus A_{2}=3$, by Corollary 2.1, the relation $\left(\mathrm{Pic}_{\Delta}\right){ }_{\Lambda_{K 3}}^{\perp} \simeq U \oplus \mathrm{Pic}_{\Delta^{\prime}}$ holds. Moreover, by Lemma 2.1, we have $\mathrm{Pic}_{\Delta} \simeq$ $\left(U^{\oplus 2} \oplus A_{2}\right)_{\Lambda_{K 3}}^{\perp} \simeq U \oplus E_{6} \oplus E_{8}$.

\subsection{No. 46}

Set one-simplices of $\Sigma$ in terms of a basis of $M_{(1,1,1,2)} \otimes \mathbb{R}$

$$
\begin{aligned}
& (-1,1,0,0),(-1,0,1,0),(-2,0,0,1) \text { : } \\
& v_{1}=(-1,-1,1), \quad v_{2}=(-1,2,0), \quad v_{3}=(2,-1,0) \text {, } \\
& v_{4}=(4,-1,-1), \quad v_{5}=(-1,4,-1), \quad v_{6}=(-1,-1,-1) \text {, } \\
& v_{7}=(-1,-1,0), \quad v_{8}=(1,0,0), \quad v_{9}=(0,1,0), \\
& v_{10}=(3,0,-1), \quad v_{11}=(2,1,-1), \quad v_{12}=(1,2,-1) \text {, } \\
& v_{13}=(0,3,-1), \quad v_{14}=(-1,3,-1), v_{15}=(-1,2,-1) \text {, } \\
& v_{16}=(-1,1,-1), v_{17}=(-1,0,-1), v_{18}=(0,-1,-1) \text {, } \\
& v_{19}=(1,-1,-1), v_{20}=(2,-1,-1), v_{21}=(3,-1,-1) \text {, }
\end{aligned}
$$

and let $\tilde{D}_{i}$ be the toric divisor determined by the lattice point $v_{i}$ for $i=1, \ldots, 21$, and $D_{i}:=\left.\tilde{D}_{i}\right|_{-K_{X}}$ with $X:=\widetilde{\mathbb{P}_{\Sigma}}$. It can be easily seen by formulas (3) and (2) that

$$
\rho_{\Delta}=21-3=18, \quad D_{1}^{2}=0, \quad D_{2}^{2}=\cdots=D_{21}^{2}=-2 .
$$

Let $L$ be a lattice generated by divisors $\left\{D_{i}\right\}_{i=1}^{21}$. By solving the Eq. (4), one sees that $\left\{D_{1}, D_{3}, D_{8}, D_{7}, D_{2}, D_{5}, D_{13}, D_{12}, D_{11}, D_{10}, D_{14}, D_{15}, D_{16}\right.$,

$\left.D_{17}, D_{6}, D_{18}, D_{19}, D_{20}\right\}$ form a basis for $L$. Since rank $L=18$ is strictly greater than 12 , the lattice $L$ is isometric to $U \oplus \tilde{L}$ with some lattice $\tilde{L}$. By a direct computation, one sees that $\operatorname{sgn} L=\left(t_{+}, t_{-}\right)=(1,17)$, discr $L=-5$, and rank $L=18$, and thus, 
$\operatorname{discr} \tilde{L}=5$ and $\operatorname{rank} \tilde{L}=16$ hold. In particular, the discriminant group $A_{L}$ of $L$ is isomorphic to $\mathbb{Z} / 5 \mathbb{Z}$, and $l\left(A_{L}\right)=1$. Therefore, one observes that

$$
19-t_{-}=2 \geq 0, \quad 3-t_{+}=2 \geq 0, \quad 22-\operatorname{rank} L=4>1=l\left(A_{L}\right)
$$

and by Corollary 2.2, $L$ is a primitive sublattice of the $K 3$ lattice. Therefore, $\mathrm{Pic}_{\Delta} \simeq$ $U \oplus \tilde{L}$ with discr $\tilde{L}=5$ and $\operatorname{rank} \tilde{L}=16$.

Set one-simplices of $\Sigma^{\prime}$ in terms of a basis of $M_{(4,5,7,9)} \otimes \mathbb{R}$

$$
\begin{aligned}
& (4,0,-1,-1),(3,-1,-1,0),(0,-1,2,-1): \\
& m_{1}=(0,0,1), m_{2}=(2,-3,-1), m_{3}=(-1,1,0), \\
& m_{4}=(0,1,0), m_{5}=(1,0,0),
\end{aligned}
$$

and let $\tilde{D}_{i}^{\prime}$ be the toric divisor determined by the lattice point $m_{i}$ for $i=1, \ldots, 5$, and $D_{i}^{\prime}:=\left.\tilde{D}_{i}^{\prime}\right|_{-K_{X}}$ with $X:=\widetilde{\mathbb{P}_{\Sigma^{\prime}}}$. It can be easily seen by formulas (3) and (2) that

$$
\rho_{\Delta^{\prime}}=5-3=2, \quad D_{1}^{\prime 2}=D_{2}^{\prime 2}=2, \quad D_{3}^{\prime 2}=10, \quad D_{4}^{\prime 2}=D_{5}^{\prime 2}=-2 .
$$

Let $L^{\prime}$ be a lattice generated by divisors $\left\{D_{i}^{\prime}\right\}_{i=1}^{5}$. By solving the Eq. (4), one sees that $\left\{D_{1}^{\prime}, D_{5}^{\prime}\right\}$ form a basis for $L$, with respect to which the intersection matrix of $L$ is given by $\left(\begin{array}{cc}2 & 1 \\ 1 & -2\end{array}\right)$. One sees that the lattice $L^{\prime}$ is a hyperbolic lattice, that is, of signature $\left(t_{+}, t_{-}\right)=(1,1)$ of $\operatorname{rank} L^{\prime}=2$ and $\operatorname{discr} L^{\prime}=-5$. In particular, the discriminant group $A_{L^{\prime}}$ of $L^{\prime}$ is isomorphic to $\mathbb{Z} / 5 \mathbb{Z}$, and $l\left(A_{L^{\prime}}\right)=1$. Therefore, one observes that

$$
19-t_{-}=18 \geq 0, \quad 3-t_{+}=2 \geq 0, \quad 22-\operatorname{rank} L^{\prime}=20>1=l\left(A_{L^{\prime}}\right)
$$

and by Corollary $2.2, L^{\prime}$ is a primitive sublattice of the $K 3$ lattice. Therefore, $\mathrm{Pic}_{\Delta^{\prime}} \simeq$ $\left(\mathbb{Z}^{2},\left(\begin{array}{cc}2 & 1 \\ 1 & -2\end{array}\right)\right)$.

Since discr Pic $\operatorname{Pin}_{\Delta}=-\operatorname{discr}^{\mathrm{Pic}_{\Delta}}=-5$, by Corollary 2.1, the relation $\left.\left(\mathrm{Pic}_{\Delta}\right)\right)_{\Lambda_{K 3}}^{\perp} \simeq$ $U \oplus \mathrm{Pic}_{\Delta^{\prime}}$ holds.

\subsection{Nos. 48 and 49}

Set one-simplices of $\Sigma^{\prime}$ in terms of a basis of $M_{(5,6,8,11)} \otimes \mathbb{R}$

$$
\begin{aligned}
& (-1,0,2,-1),(-1,-1,0,1),(5,-1,-1,-1): \\
& m_{1}=(1,0,0), m_{2}=(0,1,0), m_{3}=(0,0,1), m_{4}=(-1,3,-1)
\end{aligned}
$$

and let $\tilde{D}_{i}^{\prime}$ be the toric divisor determined by the lattice point $m_{i}$ for $i=1, \ldots, 4$, and $D_{i}^{\prime}:=\left.\tilde{D}_{i}^{\prime}\right|_{-K_{X}}$ with $X:=\widetilde{\mathbb{P}_{\Sigma^{\prime}}}$. It can be easily seen by formulas (3) and (2) that

$$
\rho_{\Delta^{\prime}}=4-3=1, \quad D_{1}^{\prime 2}=D_{2}^{\prime 2}=2, \quad D_{3}^{\prime 2}=18, \quad D_{4}^{\prime 2}=2
$$


Let $L^{\prime}$ be a lattice generated by divisors $\left\{D_{i}^{\prime}\right\}_{i=1}^{4}$. By solving the Eq. (4), one sees that $\left\{D_{1}^{\prime}\right\}$ form a basis for $L^{\prime}$. Therefore, $\operatorname{Pic}_{\Delta^{\prime}} \simeq\langle 2\rangle$. It is well-known that the lattice $(\mathbb{Z},\langle 2\rangle)$ is a primitive sublattice of the $K 3$ lattice.

Set one-simplices of $\Sigma$ in terms of a basis of $M_{(1,1,1,3)} \otimes \mathbb{R}$

$$
\begin{aligned}
& (-1,1,0,0),(-1,0,1,0),(-3,0,0,1) \text { : } \\
& v_{1}=(-1,-1,1), \quad v_{2}=(-1,-1,-1), v_{3}=(5,-1,-1) \text {, } \\
& v_{4}=(-1,-1,5), \quad v_{5}=(-1,-1,0), \quad v_{6}=(2,-1,0) \text {, } \\
& v_{7}=(-1,-1,2), \quad v_{8}=(0,-1,-1), \quad v_{9}=(1,-1,-1) \text {, } \\
& v_{10}=(2,-1,-1), v_{11}=(3,-1,-1), \quad v_{12}=(4,-1,-1) \text {, } \\
& v_{13}=(4,-1,0), \quad v_{14}=(3,-1,1), \quad v_{15}=(2,-1,2) \text {, } \\
& v_{16}=(1,-1,3), \quad v_{17}=(0,-1,4), \quad v_{18}=(-1,-1,4) \text {, } \\
& v_{19}=(-1,-1,3), v_{20}=(-1,-1,2), \quad v_{21}=(-1,-1,1) \text {, } \\
& v_{22}=(-1,-1,0) \text {, }
\end{aligned}
$$

and let $\tilde{D}_{i}$ be the toric divisor determined by the lattice point $v_{i}$ for $i=1, \ldots, 22$, and $D_{i}:=\left.\tilde{D}_{i}\right|_{-K_{X}}$ with $X:=\widetilde{\mathbb{P}_{\Sigma}}$. It can be easily seen by formulas (3) and (2) that

$$
\rho_{\Delta}=22-3=19, \quad D_{1}^{2}=0, \quad D_{2}^{2}=\cdots=D_{22}^{2}=-2 .
$$

Let $L$ be a lattice generated by divisors $\left\{D_{i}\right\}_{i=1}^{22}$. By solving the Eq. (4), one sees that $\left\{D_{21}, D_{20}, D_{19}, D_{18}, D_{4}, D_{17}, D_{16}, D_{15}, D_{14}, D_{13}, D_{3}, D_{12}, D_{11}, D_{10}, D_{9}, D_{6}, D_{1}\right.$, $\left.D_{5}, D_{2}\right\}$ form a basis for $L$. By taking a new basis

$$
\left\{\begin{array}{l}
D_{1}, D_{1}+D_{5}, D_{19}, D_{18}, D_{4}, D_{17}, D_{16}, D_{15}, D_{14}, D_{13}, D_{3}, D_{12}, \\
D_{11}, D_{10}, D_{9},-D_{1}-D_{2}-D_{5}+D_{6}, D_{21}, D_{20}, D_{2}-D_{1}
\end{array}\right\},
$$

one sees that the lattice $L$ is isometric to $U \oplus \tilde{L}$ with some lattice $\tilde{L}$.

By a direct computation, one sees that $\operatorname{sgn} L=\left(t_{+}, t_{-}\right)=(1,18), \operatorname{discr} L=2$, and $\operatorname{rank} L=19$, and thus, $\operatorname{discr} \tilde{L}=-2$ and $\operatorname{rank} \tilde{L}=17$ hold. In particular, the discriminant group $A_{L}$ of $L$ is isomorphic to $\mathbb{Z} / 2 \mathbb{Z}$, and $l\left(A_{L}\right)=1$. Therefore, one observes that

$$
19-t_{-}=1 \geq 0, \quad 3-t_{+}=2 \geq 0, \quad 22-\operatorname{rank} L=3>1=l\left(A_{L}\right)
$$

and by Corollary 2.2, $L$ is a primitive sublattice of the $K 3$ lattice. Therefore, $\mathrm{Pic}_{\Delta} \simeq$ $U \oplus \tilde{L}$ with $\operatorname{discr} \tilde{L}=-2$ and $\operatorname{rank} \tilde{L}=17$.

Since discr Pic $\operatorname{Pi}_{\Delta}=-\operatorname{discr}\left(U \oplus \operatorname{Pic}_{\Delta^{\prime}}\right)=2$, by Corollary 2.1, the relation $\left(\mathrm{Pic}_{\Delta}\right)_{\Lambda_{K 3}}^{\perp} \simeq U \oplus \mathrm{Pic}_{\Delta^{\prime}}$ holds.

Since the rank-one lattice $(\mathbb{Z},\langle 2\rangle)$ can be primitively embedded into the hyperbolic lattice $U=\langle e, f\rangle_{\mathbb{Z}}$ of rank 2 as an element $e+f$, the orthogonal complement $(\mathbb{Z},\langle 2\rangle)_{U}^{\perp}$ in $U$ is a rank-one lattice $(\mathbb{Z},\langle-2\rangle)=\langle e-f\rangle_{\mathbb{Z}}$. Therefore, we have $\mathrm{Pic}_{\Delta^{\prime}} \simeq(\mathbb{Z},\langle 2\rangle)_{U}^{\perp} \oplus U \oplus E_{8}^{\oplus 2} \simeq(\mathbb{Z},\langle-2\rangle) \oplus U \oplus E_{8}^{\oplus 2}$. 


\subsection{No. 50}

Set one-simplices of $\Sigma^{\prime}$ in terms of a basis of $M_{(7,8,9,12)} \otimes \mathbb{R}$

$$
\begin{aligned}
& (-1,2,-1,0),(-1,-1,3,-1),(-1,-1,-1,2): \\
& m_{1}=(1,0,0), m_{2}=(0,1,0), m_{3}=(0,0,1), m_{4}=(-1,-1,-1),
\end{aligned}
$$

and let $\tilde{D}_{i}^{\prime}$ be the toric divisor determined by the lattice point $m_{i}$ for $i=1, \ldots, 4$, and $D_{i}^{\prime}:=\tilde{D}_{i}^{\prime} \mid-K_{X}$ with $X:=\widetilde{\mathbb{P}_{\Sigma^{\prime}}}$. One can easily seen by formulas (3) and (2) that

$$
\rho_{\Delta}=4-3=1, \quad D_{1}^{\prime 2}=D_{2}^{\prime 2}=D_{3}^{\prime 2}=D_{4}^{\prime 2}=4
$$

Let $L^{\prime}$ be a lattice generated by divisors $\left\{D_{i}^{\prime}\right\}_{i=1}^{4}$. By solving the Eq. (4), one sees that $\left\{D_{1}^{\prime}\right\}$ form a basis for $L^{\prime}$. Therefore, $\operatorname{Pic}_{\Delta^{\prime}} \simeq(\mathbb{Z},\langle 4\rangle)$. It is well-known that the lattice $(\mathbb{Z},\langle 4\rangle)$ is a primitive sublattice of the $K 3$ lattice.

Set one-simplices of $\Sigma$ in terms of a basis of $M_{(1,1,1,1)} \otimes \mathbb{R}$

$$
\begin{aligned}
& (-1,1,0,0),(-1,0,1,0),(-1,0,0,1) \text { : } \\
& v_{1}=(-1,-1,-1), v_{2}=(3,-1,-1), \quad v_{3}=(-1,3,-1) \text {, } \\
& v_{4}=(-1,-1,3), \quad v_{5}=(0,-1,-1), \quad v_{6}=(1,-1,-1) \text {, } \\
& v_{7}=(2,-1,-1), \quad v_{8}=(-1,0,-1), \quad v_{9}=(-1,1,-1) \text {, } \\
& v_{10}=(-1,2,-1), \quad v_{11}=(-1,-1,0), v_{12}=(-1,-1,1) \text {, } \\
& v_{13}=(-1,-1,2), \quad v_{14}=(2,0,-1), \quad v_{15}=(1,1,-1) \text {, } \\
& v_{16}=(0,2,-1), \quad v_{17}=(-1,2,0), \quad v_{18}=(-1,1,1) \text {, } \\
& v_{19}=(-1,0,2), \quad v_{20}=(0,-1,2), \quad v_{21}=(1,-1,1) \text {, } \\
& v_{22}=(2,-1,0) \text {, }
\end{aligned}
$$

and let $\tilde{D}_{i}$ be the toric divisor determined by the lattice point $v_{i}$ for $i=1, \ldots, 22$, and $D_{i}:=\left.\tilde{D}_{i}\right|_{-K_{X}}$ with $X:=\widetilde{\mathbb{P}_{\Sigma}}$. It can be easily seen by formulas (3) and (2) that

$$
\rho_{\Delta}=22-3=19, \quad D_{1}^{2}=\cdots=D_{22}^{2}=-2 .
$$

Let $L$ be a lattice generated by divisors $\left\{D_{i}\right\}_{i=1}^{22}$. By solving the Eq. (4), one sees that $\left\{D_{6}, D_{5}, D_{8}, D_{1}, D_{11}, D_{12}, D_{13}, D_{4}, D_{19}, D_{18}, D_{17}, D_{3}, D_{10}, D_{16}, D_{15}, D_{14}, D_{2}\right.$, $\left.D_{22}, D_{21}\right\}$ form a basis for $L$. Since the rank of $L$ is strictly greater than 12 , the lattice $L$ is isometric to $U \oplus \tilde{L}$ with some lattice $\tilde{L}$.

By a direct computation, one sees that $\operatorname{sgn} L=\left(t_{+}, t_{-}\right)=(1,18), \operatorname{discr} L=4$, and $\operatorname{rank} L=19$, and thus, $\operatorname{discr} \tilde{L}=-4$ and $\operatorname{rank} \tilde{L}=17$ hold. In particular, the discriminant group $A_{L}$ of $L$ is isomorphic to $\mathbb{Z} / 4 \mathbb{Z}$, and $l\left(A_{L}\right)=1$. Therefore, one observes that

$$
19-t_{-}=1 \geq 0, \quad 3-t_{+}=2 \geq 0, \quad 22-\operatorname{rank} L=3>1=l\left(A_{L}\right)
$$

and by Corollary 2.2, $L$ is a primitive sublattice of the $K 3$ lattice. Therefore, $\mathrm{Pic}_{\Delta} \simeq$ $U \oplus \tilde{L}$ with discr $\tilde{L}=-4$ and $\operatorname{rank} \tilde{L}=17$. 
Since $\operatorname{discr}^{\mathrm{Pic}} \mathrm{c}_{\Delta}=-\operatorname{discr}\left(U \oplus \mathrm{Pic}_{\Delta^{\prime}}\right)=2$, by Corollary 2.1, the relation $\left.\left(\mathrm{Pic}_{\Delta}\right)\right)_{\Lambda_{K 3}}^{\perp} \simeq U \oplus \mathrm{Pic}_{\Delta^{\prime}}$ holds.

Since the rank-one lattice $(\mathbb{Z},\langle 4\rangle)$ can be primitively embedded into the hyperbolic lattice $U=\langle e, f\rangle_{\mathbb{Z}}$ of rank 2 as an element $2 e+f$, the orthogonal complement $(\mathbb{Z},\langle 4\rangle)_{U}^{\perp}$ in $U$ is a rank-one lattice $(\mathbb{Z},\langle-4\rangle)=\langle e-2 f\rangle_{\mathbb{Z}}$. Therefore, we have $\mathrm{Pic}_{\Delta^{\prime}} \simeq(\mathbb{Z},\langle 4\rangle)_{U}^{\perp} \oplus U \oplus E_{8}^{\oplus 2} \simeq(\mathbb{Z},\langle-4\rangle) \oplus U \oplus E_{8}^{\oplus 2}$.

Therefore, the assertion of Theorem 3.1 is verified.

\section{Conclusion}

We see in the main theorem that all coupling pairs that are polytope-dual with trivial toric contribution can extend to lattice duality among families of $K 3$ surfaces. Thus, the coupling is partly translated to be the lattice-duality. Moreover, all except Nos. 46, 48 and 49, and 50 admit a pair of families of $K 3$ surfaces with generic sections being elliptic: indeed, the Picard lattices $\mathrm{Pic}_{\Delta}$ and $\mathrm{Pic}_{\Delta^{\prime}}$ contain the hyperbolic lattice $U$ of rank 2 .

We can conclude that the Picard lattices of the families studied in the article are independent from the choice of reflexive polytopes. In other words, since the choice of a reflexive polytope is that of a way of blow-up of the ambient space, the Picard lattice in the subfamilies is birationally independent.

Funding Open Access funding enabled and organized by Projekt DEAL.

Open Access This article is licensed under a Creative Commons Attribution 4.0 International License, which permits use, sharing, adaptation, distribution and reproduction in any medium or format, as long as you give appropriate credit to the original author(s) and the source, provide a link to the Creative Commons licence, and indicate if changes were made. The images or other third party material in this article are included in the article's Creative Commons licence, unless indicated otherwise in a credit line to the material. If material is not included in the article's Creative Commons licence and your intended use is not permitted by statutory regulation or exceeds the permitted use, you will need to obtain permission directly from the copyright holder. To view a copy of this licence, visit http://creativecommons.org/licenses/by/4.0/.

\section{References}

Batyrev, V.V.: Dual polyhedra and mirror symmetry for Calabi-Yau hypersurfaces in toric varieties. J. Alg. Geom. 3, 493-535 (1994)

Bourbaki, N.: Groupes et algébres de Lie, chapitres 4,5, et 6. Éléments de Mathématique, Masson, Paris (1968)

Bruzzo, U., Grassi, A.: Grassi, A, Picard group of hypersurfaces in toric 3-folds. Int. J. Math. 23(2), 1-14 (2012)

Dolgachev, I.: Mirror symmetry for lattice polarized K3 surfaces. J. Math. Sci. 81, 2599-2630 (1996)

Ebeling, W.: Mirror symmetry, Kobayashis duality, and Saitos duality. Kodai Math. J. 29, 319-336 (2006)

Ebeling, W., Takahashi, A.: Strange duality of weighted homogeneous polynomials. Compos. Math. 147(5), 1413-1433 (2011)

Fulton, W.: Introduction to toric varieties. Ann. Math. Stu. 131, 2nd edn. Princeton University Press, Princeton (1997)

Kobayashi, M.: Duality of weights, mirror symmetry and Arnolds strange duality. Tokyo J. Math. 31, 225-251 (2008) 
Mase, M.: A mirror duality for families of $K 3$ surfaces associated to bimodular singularities. Manuscripta Math. 149(3-4), 389-404 (2015). https://doi.org/10.1007/s00229-015-0788-9

Mase, M.: Lattice duality for families of $K 3$ surfaces associated to transpose duality. Manuscripta Math. 155, 61-76 (2018). https://doi.org/10.1007/s00229-017-0936-5. (Online 10 April 2017)

Mase, M.: Families of $K 3$ surfaces and curves of (2, 3)-torus type. Kodai Math. J. 42(3), 409-430 (2019). https://doi.org/10.2996/kmj/1572487224

Mase, M.: Polytope duality for families of $K 3$ surfaces and coupling, Bull. Braz. Math. Soc. New Ser.52, 499-536 (2021). https://doi.org/10.1007/s00574-020-00215-8 (online 24 June 2020)

Nikulin, V.V.: Integral symmetric bilinear forms and some of their applications. Math. USSR-Izv 14, 103167 (1980)

Nishiyama, K.: The Jacobian fibrations on some $K 3$ surfaces and their Mordell-Weil groups. Jpn. J. Math. 22(2), 293-347 (1996)

Oda, T.: Torus Embeddings and Applications, Tata Institute of Fundamental Research Lectures 57. Springer, New York (1978)

Yonemura, T.: Hypersurface simple $K 3$ singularities. Tôhoku Math. J. 42, 351-380 (1990)

Publisher's Note Springer Nature remains neutral with regard to jurisdictional claims in published maps and institutional affiliations. 